\title{
Existence, uniqueness, and approximate solutions for the general nonlinear distributed-order fractional differential equations in a Banach space
}

\author{
Tahereh Eftekhari ${ }^{1}$ (D) Jalil Rashidinia ${ }^{1^{*}}$ (D) and Khosrow Maleknejad' (B)
}

"Correspondence:

rashidinia@iust.ac.ir

${ }^{1}$ School of Mathematics, Iran

University of Science \& Technology

(IUST), Narmak, Tehran, 16846

13114, Iran

\section{Springer}

\begin{abstract}
The purpose of this paper is to provide sufficient conditions for the local and global existence of solutions for the general nonlinear distributed-order fractional differential equations in the time domain. Also, we provide sufficient conditions for the uniqueness of the solutions. Furthermore, we use operational matrices for the fractional integral operator of the second kind Chebyshev wavelets and shifted fractional-order Jacobi polynomials via Gauss-Legendre quadrature formula and collocation methods to reduce the proposed equations into systems of nonlinear equations. Also, error bounds and convergence of the presented methods are investigated. In addition, the presented methods are implemented for two test problems and some famous distributed-order models, such as the model that describes the motion of the oscillator, the distributed-order fractional relaxation equation, and the Bagley-Torvik equation, to demonstrate the desired efficiency and accuracy of the proposed approaches. Comparisons between the methods proposed in this paper and the existing methods are given, which show that our numerical schemes exhibit better performances than the existing ones.
\end{abstract}

MSC: 65R20; 41A50; 41A55

Keywords: Distributed-order fractional derivative; Fixed point theorem; Operational matrices; The second kind Chebyshev wavelets; Shifted fractional-order Jacobi polynomials; Error bounds

\section{Introduction}

Distributed-order fractional derivatives indicate fractional derivatives that are integrated over the order of the differentiation within a given range [55]. In recent decades, distributed-order fractional differential equations (DOFDEs) have been used to model more phenomena in various fields such as visco-elastic [4, 6], dielectrics [10], diffusions $[11,24,26,28,40,42,49,52]$, signal processing [29], biosciences [17, 30], finance [16, 32], electrochemistry [44], and optimal control [54,56]. The motivation of DOFDEs is the generalization of single-order and multi-term fractional differential equations [27].

(c) The Author(s) 2021. This article is licensed under a Creative Commons Attribution 4.0 International License, which permits use sharing, adaptation, distribution and reproduction in any medium or format, as long as you give appropriate credit to the original author(s) and the source, provide a link to the Creative Commons licence, and indicate if changes were made. The images or other third party material in this article are included in the article's Creative Commons licence, unless indicated otherwise in a credit line to the material. If material is not included in the article's Creative Commons licence and your intended use is not permitted by statutory regulation or exceeds the permitted use, you will need to obtain permission directly from the copyright holder. To view a copy of this licence, visit http://creativecommons.org/licenses/by/4.0/ 
In [5], Atanacković et al. studied the existence and uniqueness of solutions for DOFDEs of the form

$$
\begin{aligned}
& D^{2} f(t)=\lambda \int_{0}^{2} p(q)_{0}^{C} D_{t}^{q} f(t) d q=G(t, f(t)), \quad t>0, \\
& f^{(i)}(0)=f_{0}^{(i)}, \quad i=0,1,
\end{aligned}
$$

in $L_{\text {loc }}^{1}(\mathbb{R}) \cap C^{1}([0, \infty))$. Such equations arise in distributed derivative models of system identification theory and visco-elasticity.

In this research study, we consider general nonlinear DOFDEs in the time domain $\Omega=$ $\left[0, t_{f}\right]$ as follows:

$$
\int_{\alpha}^{\beta} G_{1}\left(q,{ }_{0}^{C} D_{t}^{q} f(t)\right) d q+G_{2}\left(t, f(t),{ }_{0}^{C} D_{t}^{\alpha_{1}} f(t), \ldots,{ }_{0}^{C} D_{t}^{\alpha_{r}} f(t)\right)=g(t),
$$

with the initial conditions

$$
f^{(k)}(0)=f_{0}^{(k)}, \quad k=0,1, \ldots, \max \left\{\left\lceil\alpha_{r}\right\rceil,\lceil\beta\rceil\right\}-1 .
$$

Here, $G_{1}(\cdot)$ is a linear or nonlinear function,

$$
G_{2}\left(t, f(t),{ }_{0}^{C} D_{t}^{\alpha_{1}} f(t), \ldots,{ }_{0}^{C} D_{t}^{\alpha_{r}} f(t)\right)=\kappa_{0} f(t)+\sum_{i=1}^{r} \kappa_{i 0}^{C} D_{t}^{\alpha_{i}} f(t),
$$

where $\kappa_{i} \in \mathbb{R}$; and $\alpha, \beta, \alpha_{i}\left(\alpha_{1}<\cdots<\alpha_{r}\right)$ for $i=0,1, \ldots, r$ are positive real numbers. Also, $\lceil\beta\rceil$ denotes the ceiling function and is the smallest integer greater than or equal to $\beta$.

Note that Eq. (1) is the general form of DOFDEs in the time domain which for the case $G_{1}\left(q,{ }_{0}^{C} D_{t}^{q} f(t)\right)=\Gamma(q){ }_{0}^{C} D_{t}^{q} f(t), g(t)=0, \kappa_{j}=0, j=1, \ldots, r$, leads to the distributed-order fractional relaxation equation [31]. When $G_{1}\left(q,{ }_{0}^{C} D_{t}^{q} f(t)\right)=b \Gamma(q){ }_{0}^{C} D_{t}^{q} f(t)$ with a constant $b$, $\alpha_{1}=2, \kappa_{j}=0, j=2, \ldots, r$, Eq. (1) is the Bagley-Torvik equation [7, 8]. Also, for the case $\int_{0}^{1} a_{0}^{q} D_{t}^{q} \sigma(t) d q=\gamma \int_{0}^{1} b_{0}^{q C} D_{t}^{q} f(t) d q, \alpha_{1}=2, \kappa_{0}=\omega^{2}, \kappa_{1}=1, \kappa_{j}=0, j=2, \ldots, r$, we have the model that describes the motion of the oscillator [20], where $\gamma, a, b$ are constants; $\omega$ is the eigen frequency of the undamped system; $g(t)$ is the external forcing function; and $f(t)$, $\sigma(t)$ are the displacement and the dissipation force.

As the realm of DOFDEs describing the real-life response of physical systems grows, the demand for numerical solutions to analyze the behavior of these equations becomes more pronounced in order to overcome the mathematical complexity of analytical solutions. Therefore, the development of effective and easy-to-use numerical schemes for solving such equations acquires an increasing interest. While several numerical techniques have been proposed to solve many different problems (see, for instance, $[1-3,13,15,25,33-$ $35,43,45,48]$ and the references therein), there have been few research studies that developed numerical methods to solve general DOFDEs (see [19, 21, 36, 38, 47, 50, 53]). The development, however, for efficient numerical methods to solve DOFDEs is still an important issue [21].

The aim of this paper is to provide sufficient conditions for the existence and uniqueness of solutions for Eqs. (1) and (2). Also, we are going to approximate solutions for the 
mentioned equations with high precision. To do this, in Sect. 2, we present a review of fractional calculus, an introduction of the second kind Chebyshev wavelets (SKCWs), shifted fractional-order Jacobi polynomials (SFOJPs), function approximations, and operational matrices for the Riemann-Liouville fractional integral operator. In Sect. 3, we provide sufficient conditions for the existence and uniqueness of solutions for general DOFDEs. In Sect. 4, by using operational matrices, mentioned in Sect. 2, we approximate the solution of Eqs. (1) and (2). In Sect. 5, we obtain the error bounds for the approximations. In Sect. 6, we solve two test problems and some famous distributed-order models, such as the model that describes the motion of the oscillator, the distributed-order fractional relaxation equation, and the Bagley-Torvik equation, to show that our approaches will increase the accuracy of the methods used for such operational matrices. Finally, a conclusion is given in Sect. 7.

\section{Preliminaries}

\subsection{Fractional calculus}

Definition 2.1 ([41]) The Riemann-Liouville integral of fractional order $\iota>0$ is defined as follows:

$$
{ }_{0}^{R} I_{t}^{l} f(t)= \begin{cases}\frac{1}{\Gamma(\iota)} \int_{0}^{t}(t-\tau)^{\iota-1} f(\tau) d \tau=\frac{1}{\Gamma(\iota)} t^{\iota-1} * f(t), & \lceil\iota\rceil-1<\iota \leq\lceil\iota\rceil, \\ f(t), & \iota=0,\end{cases}
$$

where $*$ and $\Gamma(\cdot)$ are the convolution product and gamma function, respectively.

Definition 2.2 ([41]) The Caputo derivative of fractional order $\iota>0$ is defined as follows:

$$
{ }_{0}^{C} D_{t}^{\iota} f(t)= \begin{cases}\frac{1}{\Gamma(\lceil\iota\rceil-l)} \int_{0}^{t}(t-\tau)^{\lceil\iota\rceil-1-\iota} f^{(\ulcorner\iota\rceil)}(\tau) d \tau, & \lceil\iota\rceil-1<\iota<\lceil\iota\rceil, \\ f^{(\zeta \iota\rceil)}(t), & \iota=\lceil\iota\rceil,\end{cases}
$$

with the following properties:

$$
\begin{aligned}
& { }_{0}^{C} D_{t 0}^{\iota R} I_{t}^{\iota} f(t)=f(t), \\
& { }_{0}^{R} I_{t 0}^{\iota C} D_{t}^{\iota} f(t)=f(t)-\sum_{j=0}^{\lceil\iota\rceil-1} \frac{f_{0}^{(j)}}{j !} t^{j}, \\
& { }_{0}^{C} D_{t}^{\iota} f(t)={ }_{0}^{R} I_{t}^{[\iota]-\iota C} D_{t}^{[\iota]} f(t), \\
& { }_{0}^{C} D_{t 0}^{\mu R} I_{t}^{l} f(t)={ }_{0}^{R} I_{t}^{l-\mu} f(t), \quad \iota \geq \mu, \\
& { }_{0}^{C} D_{t}^{\mu} c=0 \quad \text { for constant } c, \\
& { }_{0}^{C} D_{t}^{\iota} t^{k}=\frac{\Gamma(k+1)}{\Gamma(k+1-\iota)} t^{k-\iota}, \quad k \geq\lceil\iota\rceil .
\end{aligned}
$$

Definition 2.3 ([18]) The distributed-order fractional derivative is defined as follows:

$$
{ }_{0}^{C} D_{t}^{p(v)} f(t)=\int_{v_{1}}^{v_{2}} p(v){ }_{0}^{C} D_{t}^{v} f(t) d v
$$

where $v_{1}, v_{2} \in \mathbb{R}^{+}$, and $p(v)$ is distribution of order $v \in\left[v_{1}, v_{2}\right]$. 


\subsection{SKCWs and function approximation}

SKCWs are as follows:

$$
\psi_{i, j}(t)= \begin{cases}\sqrt{\frac{2^{k+3}}{t_{f} \pi}} T_{j}^{*}\left(\frac{2^{k}}{t_{f}} t-i+1\right), & \frac{i-1}{2^{k}} t_{f} \leq t<\frac{i}{2^{k}} t_{f}, \\ 0, & \text { otherwise }\end{cases}
$$

where $i=1,2, \ldots, 2^{k}, j=0,1, \ldots, M-1$. Here, $T_{j}^{*}(t)$ is the shifted Chebyshev polynomial of the second kind of degree $j \geq 0$, defined on the interval $[0,1]$ by

$$
T_{j}^{*}(t)=\sum_{k=0}^{j} c_{k, j} t^{j-k}
$$

where

$$
c_{k, j}=\frac{(-1)^{k} 2^{2 j-2 k} \Gamma(2 j-k+2)}{\Gamma(k+1) \Gamma(2 j-2 k+2)} .
$$

Let $w(t)=\sqrt{t-t^{2}}$ be the weight function. A function $f(t) \in L_{\omega}^{2}\left(\left[0, t_{f}\right)\right)$ can be expanded by SKCWs as follows:

$$
f(t)=\sum_{i=1}^{\infty} \sum_{j=0}^{\infty} \hat{f}_{i, j} \psi_{i, j}(t)
$$

where

$$
\hat{f}_{i, j}=\int_{0}^{t_{f}} f(t) \psi_{i, j}(t) \omega(t) d t
$$

and $\omega(t)=w\left(\frac{2^{k}}{t_{f}} t-i+1\right)$. We truncate the infinite series given in Eq. (6), and then we approximate a function $f(t)$ in the following form:

$$
f(t) \simeq f_{2^{k}, M-1}(t)=\sum_{i=1}^{2^{k}} \sum_{j=0}^{M-1} \hat{f}_{i, j} \psi_{i, j}(t)=\hat{F}^{T} \Psi(t),
$$

where

$$
\begin{aligned}
& \hat{F}=\left[\hat{f}_{1,0}, \ldots, \hat{f}_{1, M-1}, \ldots, \hat{f}_{2^{k}, 0}, \ldots, \hat{f}_{2^{k}, M-1}\right]^{T}, \\
& \Psi(t)=\left[\psi_{1,0}(t), \ldots, \psi_{1, M-1}(t), \ldots, \psi_{2^{k}, 0}(t), \ldots, \psi_{2^{k}, M-1}(t)\right]^{T},
\end{aligned}
$$

are $2^{k} M \times 1$ vectors.

\subsection{SFOJPs and function approximation}

SFOJPs of order $i$ are defined on the interval $\left[0, t_{f}\right]$ by the following formula [22]:

$$
\mathcal{J}_{t f, i}^{(\lambda, \theta)}(t)=\sum_{k=0}^{i}(-1)^{i-k} \frac{\Gamma(i+\vartheta+1) \Gamma(i+k+\theta+\vartheta+1)}{\Gamma(k+\vartheta+1) \Gamma(i+\theta+\vartheta+1)(i-k) ! k ! t_{f}^{k \lambda}} t^{k \lambda},
$$

where $\theta, \vartheta \in \mathbb{R}$ and $0<\lambda<1$. 
The orthogonality property of $\mathcal{J}_{t_{f}, i}^{(\lambda, \theta, \vartheta)}(t)$ is as follows:

$$
\int_{0}^{t_{f}} \mathcal{J}_{t_{f}, i}^{(\lambda, \theta, \vartheta)}(t) \mathcal{J}_{t_{f}, i^{\prime}}^{(\lambda, \theta, \vartheta)}(t) w_{t_{f}}^{(\lambda, \theta, \vartheta)}(t) d t=h_{t_{f}, i}^{(\lambda, \theta, \vartheta)} \delta_{i i^{\prime}}
$$

where $\delta_{i i^{\prime}}$ and $w_{t_{f}}^{(\lambda, \theta, \vartheta)}(t)=\lambda t^{\lambda \vartheta+\lambda-1}\left(t_{f}^{\lambda}-t^{\lambda}\right)^{\theta}$ are Kronecker delta and weight functions, respectively. Also,

$$
h_{t_{f}, i}^{(\lambda, \theta)}=\frac{t_{f}^{(\theta+\vartheta+1) \lambda} \Gamma(i+\theta+1) \Gamma(i+\vartheta+1)}{(2 i+\theta+\vartheta+1) i ! \Gamma(i+\theta+\vartheta+1)} .
$$

By using SFOJPs, a function $f(t) \in L_{w_{t_{f}}^{(\lambda, \theta, \vartheta)}}^{2}\left(\left[0, t_{f}\right]\right)$ can be approximated as follows:

$$
f(t) \simeq f_{N}(t)=\sum_{i=0}^{N} \tilde{f}_{i} \phi_{i}(t)=\tilde{F}^{T} \Phi(t)
$$

where

$$
\tilde{f}_{i}=\frac{1}{h_{t_{f}, i}^{(\lambda, \theta, \vartheta)}} \int_{0}^{t_{f}} f(t) \phi_{i}(t) w_{t_{f}}^{(\lambda, \theta, \vartheta)}(t) d t
$$

Also, $\tilde{F}$ and $\Phi(t)$ are $(N+1) \times 1$ vectors given by

$$
\begin{aligned}
& \tilde{F}=\left[\tilde{f}_{0}, \tilde{f}_{1}, \ldots, \tilde{f}_{N}\right]^{T}, \\
& \Phi(t)=\left[\mathcal{J}_{t_{f}, 0}^{(\lambda, \theta, \vartheta)}(t), \mathcal{J}_{t_{f}, \theta}^{(\lambda, \theta, \vartheta)}(t), \ldots, \mathcal{J}_{t_{f}, N}^{(\lambda, \theta, \vartheta)}(t)\right]^{T} .
\end{aligned}
$$

\subsection{Operational matrices of the Riemann-Liouville fractional integral operator}

Following $[23,51]$, we can obtain the operational matrix of the Riemann-Liouville fractional integral operator based on SKCWs for $t \in\left[0, t_{f}\right)$ in the following theorem.

Theorem 2.1 Let $\Psi(t)$ be the vector of SKCWs. Then

$$
{ }_{0}^{R} I_{t}^{q} \Psi(t) \simeq \mathbf{I}^{q} \Psi(t)=\widehat{\Psi}(t, q)
$$

where

$$
\mathbf{I}^{q}=\Lambda \mathbf{P}^{q} \Lambda^{-1}
$$

Also,

$$
\Lambda \triangleq\left[\Psi\left(\frac{t_{f}}{2^{k+1} M}\right), \Psi\left(\frac{3 t_{f}}{2^{k+1} M}\right), \ldots, \Psi\left(\frac{\left(2^{k+1} M-1\right) t_{f}}{2^{k+1} M}\right)\right]
$$


is a $2^{k} M \times 2^{k} M$ matrix and

$$
\mathbf{P}^{q}=\left(\frac{t_{f}}{2^{k} M}\right)^{q} \frac{1}{\Gamma(q+2)}\left[\begin{array}{ccccc}
1 & \eta_{1} & \eta_{2} & \ldots & \eta_{2^{k} M-1} \\
0 & 1 & \eta_{1} & \ldots & \eta_{2^{k} M-2} \\
0 & 0 & 1 & \ldots & \eta_{2^{k} M-3} \\
\vdots & \vdots & \vdots & \ddots & \vdots \\
0 & 0 & 0 & \ldots & 1
\end{array}\right]
$$

is the operational matrix of fractional integral operator for the block-pulse functions, where

$$
\eta_{l}=(l+1)^{q+1}-2 l^{q+1}+(l-1)^{q+1}, \quad l=1,2, \ldots, 2^{k} M-1 .
$$

According to our previous work [37], we have the following theorem.

Theorem 2.2 Let $\Phi(t)$ be the vector of SFOJPs. Then

$$
{ }_{0}^{R} I_{t}^{q} \Phi(t) \simeq \mathbf{I}^{t_{f}, q} \Phi(t)=\widehat{\Phi}(t, q),
$$

where $\mathbf{I}^{t_{f}, q}$ is the operational matrix of the Riemann-Liouville integral operator of fractional order $q$ with entries

$$
\begin{aligned}
\mathbf{I}_{k l}^{t_{f}, q}= & \sum_{j=0}^{k}\left((-1)^{k-j} \frac{\Gamma(k+\vartheta+1) \Gamma(k+j+\theta+\vartheta+1) \Gamma(j \lambda+1)}{\Gamma(j+\vartheta+1) \Gamma(k+\theta+\vartheta+1)(k-j) ! j ! \Gamma(q+j \lambda+1)}\right. \\
& \left.\times \sum_{i=0}^{l}(-1)^{l-i} \frac{(2 l+\theta+\vartheta+1) l ! \Gamma(l+i+\theta+\vartheta+1) t_{f}^{q} B\left(\theta+1, i+j+\vartheta+1+\frac{q}{\lambda}\right)}{\Gamma(l+\theta+1) \Gamma(i+\vartheta+1)(l-i) ! i !}\right) .
\end{aligned}
$$

Here, $B(.,$.$) is a beta function and k, l=0,1, \ldots, N$.

\section{Existence and uniqueness of solutions}

In the following theorem, by using Schauder's fixed point theorem [57], we prove the local existence of solutions for general DOFDEs in a Banach space.

Theorem 3.1 Let $G_{1}$ be Lipschitz with the constant $\varsigma$. Suppose that

(C1) $G_{1} \in C\left(\Omega \times \mathbb{R}^{n}, \mathbb{R}^{n}\right)$ and $g, g_{1}, f, v \in C\left(\Omega, \mathbb{R}^{n}\right)$;

(C2) $\left|g(t)-g_{1}(t)\right|<\frac{\left|\kappa_{0}\right| \varepsilon}{3}$;

(C3) $\left|G_{1}\left(q,{ }_{0}^{C} D_{t}^{q} f(t)\right)-G_{1}\left(q,{ }_{0}^{C} D_{t}^{q} \nu(t)\right)\right|<\frac{\left|\kappa_{0}\right| \varepsilon}{3(\beta-\alpha)}$

(C4) $\left|{ }_{0}^{C} D_{t}^{\alpha_{i}} f(t)-{ }_{0}^{C} D_{t}^{\alpha_{i}} v(t)\right| \leq \zeta_{i}$.

Also, suppose that $\sum_{i=1}^{r}\left|\frac{\kappa_{i}}{\kappa_{0}}\right| \zeta_{i} \leq \frac{\varepsilon}{3}$. Then general DOFDEs have at least one solution on $\Omega$.

Proof Consider $D=\{(t, f): t \in \Omega,|f(t)| \leq b\}$. Suppose that $|g(t)| \leq \frac{\left|\kappa_{0}\right| b}{3},\left|G_{1}\left(q_{{ }_{0}}^{C} D_{t}^{q} f(t)\right)\right| \leq$ $\xi$, $\left|{ }_{0}^{C} D_{t}^{\alpha_{i}} f(t)\right| \leq \eta_{i}$ on $D$. Choose $\frac{(\beta-\alpha) \xi}{\left|\kappa_{0}\right|} \leq \frac{b}{3}, \sum_{i=1}^{r}\left|\frac{\kappa_{i}}{\kappa_{0}}\right| \eta_{i} \leq \frac{b}{3}$, and let $\Pi_{0}=\{f: f \in$ $\left.C\left(\Omega_{0}, \mathbb{R}^{n}\right),\|f\| \leq b\right\}$, where $\|f\|=\max _{t \in \Omega_{0}}|f(t)|$ and $\Omega_{0}=\left[0, \tau_{f}\right]$. It is clear that the set $\Pi_{0}$ is convex, closed, and bounded.

Define the operator

$$
T f(t)=\frac{1}{\kappa_{0}} g(t)-\frac{1}{\kappa_{0}} \int_{\alpha}^{\beta} G_{1}\left(q{ }_{0}^{C} D_{t}^{q} f(t)\right) d q-\sum_{i=1}^{r} \frac{\kappa_{i}}{\kappa_{0}}{ }_{0} D_{t}^{\alpha_{i}} f(t), \quad t \in \Omega_{0},
$$


for any $f \in \Pi_{0}$. Clearly, we have

$$
|T f(t)| \leq \frac{1}{\left|\kappa_{0}\right|}|g(t)|+\frac{1}{\left|\kappa_{0}\right|} \int_{\alpha}^{\beta}\left|G_{1}\left(q,{ }_{0}^{C} D_{t}^{q} f(t)\right)\right| d q+\sum_{i=1}^{r}\left|\frac{\kappa_{i}}{\kappa_{0}}\right|{ }_{0}^{C} D_{t}^{\alpha_{i}} f(t) \mid \leq b .
$$

Therefore, $\|T f\| \leq b$, and we can deduce $T\left(\Pi_{0}\right) \subset \Pi_{0}$. Furthermore, for any $t_{1}, t_{2} \in \Omega_{0}$ such that $t_{2}>t_{1}$, we have

$$
\begin{aligned}
T f\left(t_{2}\right)-T f\left(t_{1}\right)= & \frac{1}{\kappa_{0}}\left(g\left(t_{2}\right)-g\left(t_{1}\right)\right)-\frac{1}{\kappa_{0}} \int_{\alpha}^{\beta}\left(G_{1}\left(q,{ }_{0}^{C} D_{t}^{q} f\left(t_{2}\right)\right)-G_{1}\left(q,{ }_{0}^{C} D_{t}^{q} f\left(t_{1}\right)\right)\right) d q \\
& -\sum_{i=1}^{r} \frac{\kappa_{i}}{\kappa_{0}}\left({ }_{0}^{C} D_{t}^{\alpha_{i}} f\left(t_{2}\right)-{ }_{0}^{C} D_{t}^{\alpha_{i}} f\left(t_{1}\right)\right) .
\end{aligned}
$$

Since $G_{1}$ is Lipschitz with the constant $\varsigma$, we get

$$
\left|G_{1}\left(q,{ }_{0}^{C} D_{t}^{q} f\left(t_{2}\right)\right)-G_{1}\left(q,{ }_{0}^{C} D_{t}^{q} f\left(t_{1}\right)\right)\right| \leq \varsigma\left|{ }_{0}^{C} D_{t}^{q} f\left(t_{2}\right)-{ }_{0}^{C} D_{t}^{q} f\left(t_{1}\right)\right| .
$$

Now we can write

$$
\begin{aligned}
\left|T f\left(t_{2}\right)-T f\left(t_{1}\right)\right| & \\
\leq & \frac{1}{\left|\kappa_{0}\right|}\left|g\left(t_{2}\right)-g\left(t_{1}\right)\right|+\frac{1}{\left|\kappa_{0}\right|} \int_{\alpha}^{\beta}\left|G_{1}\left(q,{ }_{0}^{C} D_{t}^{q} f\left(t_{2}\right)\right)-G_{1}\left(q,{ }_{0}^{C} D_{t}^{q} f\left(t_{1}\right)\right)\right| d q \\
& +\sum_{i=1}^{r}\left|\frac{\kappa_{i}}{\kappa_{0}}\right|\left|{ }_{0}^{C} D_{t}^{\alpha_{i}} f\left(t_{2}\right)-{ }_{0}^{C} D_{t}^{\alpha_{i}} f\left(t_{1}\right)\right| \\
\leq & \frac{1}{\left|\kappa_{0}\right|}\left|g\left(t_{2}\right)-g\left(t_{1}\right)\right|+\frac{\varsigma(\beta-\alpha)}{\left|\kappa_{0}\right|}\left|{ }_{0}^{C} D_{t}^{q} f\left(t_{2}\right)-{ }_{0}^{C} D_{t}^{q} f\left(t_{1}\right)\right| \\
& +\sum_{i=1}^{r}\left|\frac{\kappa_{i}}{\kappa_{0}}\right|\left|{ }_{0}^{C} D_{t}^{\alpha_{i}} f\left(t_{2}\right)-{ }_{0}^{C} D_{t}^{\alpha_{i}} f\left(t_{1}\right)\right| .
\end{aligned}
$$

Note that if $t_{2} \rightarrow t_{1}$, then the right-hand side of (10) tends to zero. Therefore, $T: \Pi_{0} \rightarrow \Pi_{0}$ is equicontinuous, and consequently, from the Arzela-Ascoli theorem [12], the closure of $T\left(\Pi_{0}\right)$ is compact.

Let

$$
T v(t)=\frac{1}{\kappa_{0}} g_{1}(t)-\frac{1}{\kappa_{0}} \int_{\alpha}^{\beta} G_{1}\left(q,{ }_{0}^{C} D_{t}^{q} \nu(t)\right) d q-\sum_{i=1}^{r} \frac{\kappa_{i}}{\kappa_{0}}{ }_{0}^{C} D_{t}^{\alpha_{i}} v(t),
$$

where $v \in \Pi_{0}$. We need to show that $T$ is continuous. Clearly, we have

$$
\begin{aligned}
|T f(t)-T \nu(t)| \leq & \frac{1}{\left|\kappa_{0}\right|}\left|g(t)-g_{1}(t)\right|+\frac{1}{\left|\kappa_{0}\right|} \int_{\alpha}^{\beta}\left|G_{1}\left(q,{ }_{0}^{C} D_{t}^{q} f(t)\right)-G_{1}\left(q_{{ }_{0}}^{C} D_{t}^{q} \nu(t)\right)\right| d q \\
& +\sum_{i=1}^{r}\left|\frac{\kappa_{i}}{\kappa_{0}}\right|\left|{ }_{0}^{C} D_{t}^{\alpha_{i}} f(t)-{ }_{0}^{C} D_{t}^{\alpha_{i}} v(t)\right| .
\end{aligned}
$$


Suppose that, for any $\varepsilon>0$, there exists $\delta>0$ such that $|f(t)-v(t)|<\delta$. Assume that assumptions $(\mathrm{C} 1)-(\mathrm{C} 4)$ hold, therefore

$$
|T f(t)-T v(t)| \leq \varepsilon
$$

and the proof is completed.

Now, by using Tychonoff's fixed point theorem [57], we are going to discuss a global existence result for general DOFDEs.

\section{Theorem 3.2 Assume that}

(D1) $G_{1} \in C\left(\mathbb{R}_{+} \times \mathbb{R}^{n}, \mathbb{R}^{n}\right)$ and $K \in C\left(\mathbb{R}_{+}^{2}, \mathbb{R}^{n}\right)$;

(D2) $K\left(q,{ }_{0}^{C} D_{t}^{q} u(t)\right)$ is monotone nondecreasing in $u$ for each $t \in \mathbb{R}_{+}$;

(D3) $\left|G_{1}\left(q,{ }_{0}^{C} D_{t}^{q} f\right)\right| \leq K\left(q,{ }_{0}^{C} D_{t}^{q} f \mid\right)$ for $\left(q,{ }_{0}^{C} D_{t}^{q} f\right) \in \mathbb{R}_{+} \times \mathbb{R}^{n}$;

(D4) $\left|{ }_{0}^{C} D_{t}^{q} f(t)\right| \leq{ }_{0}^{C} D_{t}^{q} u(t)$ and $\left|{ }_{0}^{C} D_{t}^{\alpha_{i}} f(t)\right| \leq{ }_{0}^{C} D_{t}^{\alpha_{i}} u(t)$ for $i=1,2, \ldots, r$.

Then the following DOFDE

$$
u(t)=\frac{1}{\kappa_{0}^{\prime}} x(t)+\frac{1}{\kappa_{0}} \int_{\alpha}^{\beta} K\left(q,{ }_{0}^{C} D_{t}^{q} u(t)\right) d q+\sum_{i=1}^{r} \frac{\kappa_{i}^{\prime}}{\kappa_{0}^{\prime}} D_{t}^{\alpha_{i}} u(t)
$$

has a solution $u(t)$ which exists for every $t \geq 0$, where $\kappa_{0}^{\prime} \leq\left|\kappa_{0}\right|$ and $\left|\kappa_{i}\right| \leq \kappa_{i}^{\prime}$ for $i=1,2, \ldots, r$. Also, then for every $x(t) \in \mathbb{R}_{+}$such that $|g(t)| \leq x(t)$, there exists a solution $f(t)$ for $E q$. (1) which satisfies $|f(t)| \leq u(t)$.

Proof Let $\mathcal{V}$ be a real space of all continuous functions from $(0, \infty)$ into $\mathbb{R}^{n}$. The topology on $\mathcal{V}$ being that induced by the family of pseudo-norms $\left\{\mathcal{V}_{m}(f)\right\}_{m=1}^{\infty}$, where $\mathcal{V}_{m}(f)=$ $\sup _{0 \leq t \leq m}|f(t)|$, for $f \in \mathcal{V}$. Let $\left\{\mathcal{S}_{m}\right\}_{m=1}^{\infty}$ be a set of neighborhoods, where $\mathcal{S}_{m}=\{f \in \mathcal{V}$ : $\left.\mathcal{V}_{m}(f) \leq 1\right\}$. Under this topology, $\mathcal{V}$ is a linear space, locally convex and complete.

Now consider

$$
\mathcal{V}_{0}=\{f \in \mathcal{V}:|f(t)| \leq u(t), t \geq 0\} \subseteq \mathcal{V}
$$

where $u(t)$ is a solution of (11). Clearly, in the topology of $\mathcal{V}, \mathcal{V}_{0}$ is bounded, convex, and closed.

Consider (11) whose fixed point corresponds to a solution of (1). Evidently, in the topology of $\mathcal{V}$, the map $T$ is compact. Hence, in view of the boundedness of $\mathcal{V}_{0}$, the closure of $T\left(\mathcal{V}_{0}\right)$ is compact.

Considering assumptions (D1)-(D4) yields

$$
\begin{aligned}
|T f(t)| & \leq \frac{1}{\left|\kappa_{0}\right|}|g(t)|+\frac{1}{\left|\kappa_{0}\right|} \int_{\alpha}^{\beta}\left|G_{1}\left(q,{ }_{0}^{C} D_{t}^{q} f(t)\right)\right| d q+\sum_{i=1}^{r}\left|\frac{\kappa_{i}}{\kappa_{0}}\right|\left|{ }_{0}^{C} D_{t}^{\alpha_{i}} f(t)\right| \\
& \leq \frac{1}{\left|\kappa_{0}\right|}|g(t)|+\frac{1}{\left|\kappa_{0}\right|} \int_{\alpha}^{\beta} K\left(q,\left|{ }_{0}^{C} D_{t}^{q} f(t)\right|\right) d q+\sum_{i=1}^{r}\left|\frac{\kappa_{i}}{\kappa_{0}}\right|\left|{ }_{0}^{C} D_{t}^{\alpha_{i}} f(t)\right| \\
& \leq \frac{1}{\kappa_{0}^{\prime}} x(t)+\frac{1}{\kappa_{0}^{\prime}} \int_{\alpha}^{\beta} K\left(q,{ }_{0}^{C} D_{t}^{q} u(t)\right) d q+\sum_{i=1}^{r} \frac{\kappa_{i}^{\prime}}{\kappa_{0}^{\prime}} D_{t}^{\alpha_{i}} u(t)=u(t) .
\end{aligned}
$$


Since $u(t)$ is a solution of $(11)$, using the definition of $\mathcal{V}_{0}$ gives $|T f(t)| \leq u(t)$. Thus, $T\left(\mathcal{V}_{0}\right) \subset$ $\mathcal{V}_{0}$, and from Tychonoff's fixed point theorem [57], $T$ has a fixed point in $\mathcal{V}_{0}$. Therefore, the proof of this theorem is completed.

Now we are going to prove the uniqueness of the solution for general DOFDEs.

Theorem 3.3 Let $G_{1} \in C\left(\mathbb{R} \times \mathbb{R}^{n}, \mathbb{R}^{n}\right), f \in C\left(\Omega, \mathbb{R}^{n}\right)$. Assume that there exists $0<L_{j}<1$ $(j=0,1, \ldots, r)$ such that

$$
\begin{aligned}
& \left|G_{1}\left(q,{ }_{0}^{C} D_{t}^{q} f(t)\right)-G_{1}\left(q,{ }_{0}^{C} D_{t}^{q} f_{1}(t)\right)\right| \leq L_{0}\left|f(t)-f_{1}(t)\right|, \\
& \left|{ }_{0}^{C} D_{t}^{\alpha_{i}} f(t)-{ }_{0}^{C} D_{t}^{\alpha_{i}} f_{1}(t)\right| \leq L_{i}\left|f(t)-f_{1}(t)\right| .
\end{aligned}
$$

If $\left(\frac{L_{0}(\beta-\alpha)}{\left|\kappa_{0}\right|}+\sum_{i=1}^{r}\left|\frac{\kappa_{i}}{\kappa_{0}}\right| L_{i}\right)<1$, then the general DOFDE has a unique solution.

Proof Let

$$
T f_{1}(t)=\frac{1}{\kappa_{0}} g(t)-\frac{1}{\kappa_{0}} \int_{\alpha}^{\beta} G_{1}\left(q,{ }_{0}^{C} D_{t}^{q} f_{1}(t)\right) d q-\sum_{i=1}^{r} \frac{\kappa_{i}}{\kappa_{0}}{ }_{0}^{C} D_{t}^{\alpha_{i}} f_{1}(t), \quad t \in \Omega
$$

Then we have

$$
\begin{aligned}
\left|T f(t)-T f_{1}(t)\right| \leq & \frac{1}{\left|\kappa_{0}\right|} \int_{\alpha}^{\beta}\left|G_{1}\left(q,{ }_{0}^{C} D_{t}^{q} f(t)\right)-G_{1}\left(q,{ }_{0}^{C} D_{t}^{q} f_{1}(t)\right)\right| d q \\
& +\sum_{i=1}^{r}\left|\frac{\kappa_{i}}{\kappa_{0}}\right|\left|{ }_{0}^{C} D_{t}^{\alpha_{i}} f(t)-{ }_{0}^{C} D_{t}^{\alpha_{i}} f_{1}(t)\right| \\
\leq & \frac{L_{0}(\beta-\alpha)}{\left|\kappa_{0}\right|}\left|f(t)-f_{1}(t)\right|+\sum_{i=1}^{r}\left|\frac{\kappa_{i}}{\kappa_{0}}\right| L_{i}\left|f(t)-f_{1}(t)\right| \\
& <\left(\frac{L_{0}(\beta-\alpha)}{\left|\kappa_{0}\right|}+\sum_{i=1}^{r}\left|\frac{\kappa_{i}}{\kappa_{0}}\right| L_{i}\right)\left|f(t)-f_{1}(t)\right|
\end{aligned}
$$

for any $t \in \Omega$ and $f, f_{1} \in C\left(\Omega, \mathbb{R}^{n}\right)$. Therefore,

$$
\left\|T f(t)-T f_{1}(t)\right\| \leq\left(\frac{L_{0}(\beta-\alpha)}{\left|\kappa_{0}\right|}+\sum_{i=1}^{r}\left|\frac{\kappa_{i}}{\kappa_{0}}\right| L_{i}\right)\left\|f-f_{1}\right\|
$$

Since $\left(\frac{L_{0}(\beta-\alpha)}{\left|\kappa_{0}\right|}+\sum_{i=1}^{r}\left|\frac{\kappa_{i}}{\kappa_{0}}\right| L_{i}\right)<1$, then $T$ is a contraction map in $C\left(\Omega, \mathbb{R}^{n}\right)$. Consequently, it has a unique fixed point, and therefore the general DOFDE has a unique solution $f \in$ $C\left(\Omega, \mathbb{R}^{n}\right)$.

\section{The methods of solution}

\subsection{Explanation of the SKCWs method}

In this section, without loss of generality we suppose that $\beta \geq \alpha_{r}$. Now we approximate ${ }_{0}^{C} D_{t}^{\beta} f(t)$ by the SKCWs as follows:

$$
{ }_{0}^{C} D_{t}^{\beta} f(t) \simeq \hat{F}^{T} \Psi(t) .
$$


By applying Eqs. (3) and (9), one can obtain

$$
f(t) \simeq \hat{F}^{T} \widehat{\Psi}(t, \beta)+\sum_{j=0}^{\lceil\beta\rceil-1} \frac{f_{0}^{(j)}}{j !} t^{j}
$$

Now we take the operator ${ }_{0}^{C} D_{t}^{q}$ of Eq. (12). So, we have

$$
{ }_{0}^{C} D_{t}^{q} f(t) \simeq \hat{F}^{T} \widehat{\Psi}(t, \beta-q)+\sum_{j=0}^{\lceil\beta\rceil-1} \frac{f_{0}^{(j)}}{j_{j}{ }_{0}^{C}} D_{t}^{q}\left(t^{j}\right)
$$

Note that from properties (4) and (5), ${ }_{0}^{C} D_{t}^{q}\left(t^{j}\right)$ can be determined.

Also, we take the operator ${ }_{0}^{C} D_{t}^{\alpha_{i}}, i=1,2, \ldots, r$, of Eq. (12). Using the obtained results in (1) gives

$$
\begin{aligned}
& \int_{\alpha}^{\beta} G_{1}\left(q, \hat{F}^{T} \widehat{\Psi}(t, \beta-q)+\sum_{j=0}^{\lceil\beta\rceil-1} \frac{f_{0}^{(j)}}{j !}{ }_{0}^{C} D_{t}^{q}\left(t^{j}\right)\right) d q+G_{2}\left(t, \hat{F}^{T} \widehat{\Psi}(t, \beta)\right. \\
& +\sum_{j=0}^{\lceil\beta\rceil-1} \frac{f_{0}^{(j)}}{j !} t^{j}, \hat{F}^{T} \widehat{\Psi}\left(t, \beta-\alpha_{1}\right)+\sum_{j=0}^{\lceil\beta\rceil-1} \frac{f_{0}^{(j)}{ }_{j !}^{C} D_{t}^{\alpha_{1}}\left(t^{j}\right), \ldots, \hat{F}^{T} \widehat{\Psi}\left(t, \beta-\alpha_{r}\right)}{+\lceil\beta\rceil-1} \sum_{j=0}^{(j)} \frac{\left.f_{0}{ }_{j}^{C} D^{\alpha_{r}}\left(t^{j}\right)\right) \simeq g(t) .}{}
\end{aligned}
$$

Using the Gauss-Legendre formula and collocating the obtained equation at

$$
t_{m}=\frac{(m-0.5) t_{f}}{2^{k} M}, \quad m=1,2, \ldots, 2^{k} M
$$

leads to

$$
\begin{aligned}
& \sum_{n=1}^{N^{\prime}} \frac{\beta-\alpha}{2} w_{n} G_{1}\left(\frac{\beta+\alpha}{2}+\frac{\beta-\alpha}{2} \tau_{n}, \hat{F}^{T} \widehat{\Psi}\left(t_{m}, \beta-\left(\frac{\beta+\alpha}{2}+\frac{\beta-\alpha}{2} \tau_{n}\right)\right)\right. \\
& \left.\quad+\sum_{j=0}^{\lceil\beta\rceil-1} \frac{f_{0}^{(j)}}{j !}\left({ }_{0}^{C} D_{t}^{\left(\frac{\beta+\alpha}{2}+\frac{\beta-\alpha}{2} \tau_{n}\right)}\left(t^{j}\right)\right)_{t=t_{m}}\right)+G_{2}\left(t_{m}, \hat{F}^{T} \widehat{\Psi}\left(t_{m}, \beta\right)\right. \\
& \quad+\sum_{j=0}^{\lceil\beta\rceil-1} \frac{f_{0}^{(j)}}{j !} t_{m}^{j}, \hat{F}^{T} \widehat{\Psi}\left(t_{m}, \beta-\alpha_{1}\right)+\sum_{j=0}^{\lceil\beta\rceil-1} \frac{f_{0}^{(j)}}{j !}\left({ }_{0}^{C} D_{t}^{\alpha_{1}}\left(t^{j}\right)\right)_{t=t_{m}}, \ldots, \hat{F}^{T} \widehat{\Psi}\left(t_{m}, \beta-\alpha_{r}\right) \\
& \left.\quad+\sum_{j=0}^{\lceil\beta\rceil-1} \frac{f_{0}^{(j)}}{j !}\left({ }_{0}^{C} D_{t}^{\alpha_{r}}\left(t^{j}\right)\right)_{t=t_{m}}\right) \simeq g\left(t_{m}\right),
\end{aligned}
$$

where $w_{n}$ and $\tau_{n}$ are weights and nods of Gauss-Legendre quadrature rule [9], respectively. By using the "fsolve" command of Maple 2018, we solve the arising system, and then we determine $\hat{F}$. Finally, from Eq. (7), an approximate solution for Eqs. (1) and (2) can be obtained. 


\subsection{Explanation of the SFOJPs method}

Now similar to Sect. 4.1, by using SFOJPs, we convert Eqs. (1) and (2) to a system of equations as follows:

$$
\begin{aligned}
& \sum_{n^{\prime}=1}^{N^{\prime \prime}} \frac{\beta-\alpha}{2} w_{n^{\prime}} G_{1}\left(\frac{\beta+\alpha}{2}+\frac{\beta-\alpha}{2} \tau_{n^{\prime}}, \tilde{F}^{T} \widehat{\Phi}\left(t_{m^{\prime}}, \beta-\left(\frac{\beta+\alpha}{2}+\frac{\beta-\alpha}{2} \tau_{n^{\prime}}\right)\right)\right. \\
& \left.\quad+\sum_{j=0}^{\lceil\beta\rceil-1} \frac{f_{0}^{(j)}}{j !}\left({ }_{0}^{C} D_{t}^{\left(\frac{\beta+\alpha}{2}+\frac{\beta-\alpha}{2} \tau_{n^{\prime}}\right)}\left(t^{j}\right)\right)_{t=t_{m^{\prime}}}\right)+G_{2}\left(t_{m^{\prime}}, \tilde{F}^{T} \widehat{\Phi}\left(t_{m^{\prime}}, \beta\right)\right. \\
& \quad+\sum_{j=0}^{\lceil\beta\rceil-1} \frac{f_{0}^{(j)}}{j !} t_{m^{\prime}}^{j}, \tilde{F}^{T} \widehat{\Phi}\left(t_{m^{\prime}}, \beta-\alpha_{1}\right)+\sum_{j=0}^{\lceil\beta\rceil-1} \frac{f_{0}^{(j)}}{j !}\left({ }_{0}^{C} D_{t}^{\alpha_{1}}\left(t^{j}\right)\right)_{t=t_{m^{\prime}}}, \ldots, \tilde{F}^{T} \widehat{\Phi}\left(t_{m^{\prime}}, \beta-\alpha_{r}\right) \\
& \left.\quad+\sum_{j=0}^{\lceil\beta\rceil-1} \frac{f_{0}^{(j)}}{j !}\left({ }_{0}^{C} D_{t}^{\alpha_{r}}\left(t^{j}\right)\right)_{t=t_{m^{\prime}}}\right) \simeq g\left(t_{m^{\prime}}\right),
\end{aligned}
$$

where $t_{m^{\prime}}, m^{\prime}=0,1, \ldots, N$, are roots of SFOJPs. Also, $w_{n^{\prime}}$ and $\tau_{n^{\prime}}$ are weights and nods of Gauss-Legendre quadrature rule [9], respectively. By the "fsolve" command of Maple 2018, we solve the above system, and then the unknown vector $\tilde{F}$ can be determined. Finally, from Eq. (8), we obtain an approximate solution for Eqs. (1) and (2).

\section{Error bounds}

\subsection{Error bounds for the SKCWs method}

In this subsection, we present error bounds for the SKCWs method. To do this, we define

$$
\begin{aligned}
& \langle u, v\rangle_{\omega}=\int_{0}^{t_{f}} u(t) v(t) \omega(t) d t, \quad \forall u, v \in L_{\omega}^{2}\left(\left[0, t_{f}\right)\right), \\
& \|u\|_{\omega}=\left(\int_{0}^{t_{f}} u^{2}(t) \omega(t) d t\right)^{\frac{1}{2}}, \quad \forall u \in L_{\omega}^{2}\left(\left[0, t_{f}\right)\right),
\end{aligned}
$$

which are inner product and norm on the space $L_{\omega}^{2}\left(\left[0, t_{f}\right)\right)$, respectively.

Now we recall the following theorems from our previous work [47].

Theorem 5.1 Let $f(t) \in L_{\omega}^{2}\left(\left[0, t_{f}\right)\right)$ with $\left|f^{\prime \prime}(t)\right| \leq \mathfrak{L}$. The Eq. (7) converges uniformly to $f(t)$ and the coefficients in (6) explicitly satisfy

$$
\left|\hat{f}_{i, j}\right|<4 \sqrt{2 t_{f} \pi} \mathfrak{L} \frac{1}{i^{\frac{5}{2}}(j+1)^{2}}, \quad i \geq 1, j \geq 0 .
$$

Theorem 5.2 Let $f(t) \in L_{\omega}^{2}\left(\left[0, t_{f}\right)\right)$. Then we have

$$
\left\|f-f_{2^{k}, M-1}\right\|_{\omega}<4 \sqrt{2 t_{f} \pi} \mathfrak{L}\left(\sum_{i=0}^{\infty} \sum_{j=M}^{\infty} \frac{1}{i^{5}(j+1)^{4}}+\sum_{i=2^{k}+1}^{\infty} \sum_{j=0}^{\infty} \frac{1}{i^{5}(j+1)^{4}}\right)^{\frac{1}{2}} .
$$


Theorem 5.3 Let ${ }_{0}^{C} D_{t}^{\alpha_{l}} f(t) \in L_{\omega}^{2}\left(\left[0, t_{f}\right)\right)$ and $\left|{ }_{0}^{C} D_{t}^{\alpha_{l}+2} f(t)\right| \leq \mathfrak{L}_{l}$ for $l=1,2, \ldots, r$. Then we have

$$
\left\|{ }_{0}^{C} D_{t}^{\alpha_{l}} f-\left({ }_{0}^{C} D_{t}^{\alpha_{l}} f\right)_{2^{k}, M-1}\right\|_{\omega}<4 \sqrt{2 t_{f} \pi} \mathfrak{L}_{l}\left(\sum_{i=0}^{\infty} \sum_{j=M}^{\infty} \frac{1}{i^{5}(j+1)^{4}}+\sum_{i=2^{k}+1}^{\infty} \sum_{j=0}^{\infty} \frac{1}{i^{5}(j+1)^{4}}\right)^{\frac{1}{2}} .
$$

\subsection{Error bounds for the SFOJPs method}

Here, we discuss error bounds for the SFOJPs method. To do this, first, we define the following inner product and norm on the weighted space $L_{w_{t_{f}}^{(\lambda, \theta)}}^{2}\left(\left[0, t_{f}\right]\right)$ :

$$
\begin{aligned}
& \langle u, v\rangle_{w_{t_{f}}^{(\lambda, \theta, \vartheta)}}=\int_{0}^{t_{f}} u(t) v(t) w_{t_{f}}^{(\lambda, \theta, \vartheta)}(t) d t, \quad \forall u, v \in L_{w_{t_{f}}^{(\lambda, \theta, \vartheta)}}^{2}\left(\left[0, t_{f}\right]\right), \\
& \|u\|_{w_{t_{f}}^{(\lambda, \theta, \vartheta)}}=\left(\int_{0}^{t_{f}} u^{2}(t) w_{t_{f}}^{(\lambda, \theta, \vartheta)}(t) d t\right)^{\frac{1}{2}}, \quad \forall u \in L_{w_{t_{f}}^{(\lambda, \theta, \vartheta)}}^{2}\left(\left[0, t_{f}\right]\right) .
\end{aligned}
$$

Let

$$
\Lambda_{N}=\operatorname{span}\left\{\mathcal{J}_{i}^{(\lambda, \theta, \vartheta)}(t), 0 \leq i \leq N\right\}
$$

be the fractional-polynomial space of finite dimension.

Theorem 5.4 $\operatorname{Let}_{0}^{C} D_{t}^{j \lambda} f(t) \in C\left(\left[0, t_{f}\right]\right)$, for $j=0,1, \ldots, N$. If $f_{N}(t)$ is the best approximation to $f(t)$ from $\Lambda_{N}$, then

$$
\left\|f-f_{N}\right\|_{w_{t_{f}}^{(\lambda, \theta, \vartheta)}} \leq \frac{\mathcal{L}}{\Gamma((N+1) \lambda+1)} \sqrt{\frac{t_{f}^{(2 N+3+\vartheta+\theta) \lambda} \Gamma(1+\theta) \Gamma(2 N+3+\vartheta)}{\Gamma(4+2 N+\theta+\vartheta)}},
$$

where $\mathcal{L} \geq\left|{ }_{0}^{C} D_{t}^{(N+1) \lambda} f(t)\right|$, for $t \in\left[0, t_{f}\right]$.

Proof Since $f_{N}(t)$ is the best approximation to $f(t)$ from $\Lambda_{N}$, defined in (13), we have

$$
\left\|f-f_{N}\right\|_{w_{t_{f}}^{(\lambda, \theta, \vartheta)}} \leq\|f-u\|_{w_{t_{f}}^{(\lambda, \theta)}}, \quad \forall u(t) \in \Lambda_{N}
$$

Considering the generalized Taylors formula $u(t)=\sum_{j=0}^{N} \frac{t^{j \lambda}}{\Gamma(j \lambda+1)}\left({ }_{0}^{C} D_{t}^{j \lambda} u\right)\left(0^{+}\right)$yields

$$
|f(t)-u(t)|=\left|f(t)-\sum_{j=0}^{N} \frac{t^{j \lambda}}{\Gamma(j \lambda+1)}\left({ }_{0}^{C} D_{t}^{j \lambda} u\right)\left(0^{+}\right)\right| \leq \mathcal{L} \frac{t^{(N+1) \lambda}}{\Gamma((N+1) \lambda+1)} .
$$

Taking $L_{w_{t_{f}}^{(\lambda, \theta)}}^{2(\lambda, \vartheta)}$-norm in both sides of inequality (15) leads to

$$
\begin{aligned}
\|f-u\|_{w_{t_{f}}^{(\lambda, \theta, \vartheta)}}^{2} & \leq \frac{\mathcal{L}^{2}}{(\Gamma((N+1) \lambda+1))^{2}} \int_{0}^{t_{f}} t^{2(N+1) \lambda} w_{t_{f}}^{(\lambda, \theta, \vartheta)} d t \\
& =\frac{\mathcal{L}^{2}}{(\Gamma((N+1) \lambda+1))^{2}} \lambda \int_{0}^{t_{f}} t^{\lambda(2 N+3+\vartheta)-1}\left(t_{f}^{\lambda}-t^{\lambda}\right)^{\theta} d t .
\end{aligned}
$$


Let $z=t_{f}^{\lambda}-t^{\lambda}$. Then we obtain

$$
\lambda \int_{0}^{t_{f}} t^{\lambda(2 N+3+\vartheta)-1}\left(t_{f}^{\lambda}-t^{\lambda}\right)^{\theta} d t=\int_{0}^{t_{f}^{\lambda}}\left(t_{f}^{\lambda}-z\right)^{2 N+2+\vartheta} z^{\theta} d z
$$

Now setting $s=\frac{z}{t_{f}^{\lambda}}$ gives

$$
\begin{aligned}
\int_{0}^{t_{f}^{\lambda}}\left(t_{f}^{\lambda}-z\right)^{2 N+2+\vartheta} z^{\theta} d z & =t_{f}^{(2 N+3+\vartheta+\theta) \lambda} \int_{0}^{1}(1-s)^{2 N+2+\vartheta} s^{\theta} d s \\
& =t_{f}^{(2 N+3+\vartheta+\theta) \lambda} \frac{\Gamma(1+\theta) \Gamma(2 N+3+\vartheta)}{\Gamma(4+2 N+\theta+\vartheta)} .
\end{aligned}
$$

By substituting the above relation into (17) and from (16), we can write

$$
\|f-u\|_{w_{t_{f}}^{(\lambda, \theta, \vartheta)}}^{2} \leq \frac{\mathcal{L}^{2}}{(\Gamma((N+1) \lambda+1))^{2}} t_{f}^{(2 N+3+\vartheta+\theta) \lambda} \frac{\Gamma(1+\theta) \Gamma(2 N+3+\vartheta)}{\Gamma(4+2 N+\theta+\vartheta)} .
$$

By taking the square root of (19), we obtain inequality (14).

Theorem 5.5 Let ${ }_{0}^{C} D_{t}^{j \lambda+\alpha_{l}} f(t) \in C\left(\left[0, t_{f}\right]\right)$. If $\left({ }_{0}^{C} D_{t}^{\alpha_{l}} f\right)_{N}(t)$ is the best approximation to ${ }_{0}^{C} D_{t}^{\alpha_{l}} f(t)$ from $\Lambda_{N}$, then

$$
\begin{aligned}
& \left\|{ }_{0}^{C} D_{t}^{\alpha_{l}} f-\left({ }_{0}^{C} D_{t}^{\alpha_{l}} f\right)_{N}\right\|_{w_{t_{f}}^{(\lambda, \theta, \vartheta)}} \\
& \leq \frac{\mathcal{L}_{l}}{\Gamma((N+1) \lambda+1)} \sqrt{\frac{t_{f}^{(2 N+3+\vartheta+\theta) \lambda} \Gamma(1+\theta) \Gamma(2 N+3+\vartheta)}{\Gamma(4+2 N+\theta+\vartheta)}},
\end{aligned}
$$

where $\mathcal{L}_{l} \geq{ }_{0}^{C} D_{t}^{(N+1) \lambda+\alpha_{l}} f(t) \mid$, for $l=1,2, \ldots, r$ and $t \in\left[0, t_{f}\right]$.

Proof Since $\left({ }_{0}^{C} D_{t}^{\alpha_{l}} f\right)_{N}(t)$ is the best approximation to ${ }_{0}^{C} D_{t}^{\alpha_{l}} f(t)$ from $\Lambda_{N}$, we have

$$
\left\|{ }_{0}^{C} D_{t}^{\alpha_{l}} f-\left({ }_{0}^{C} D_{t}^{\alpha_{l}} f\right)_{N}\right\|_{w_{t_{f}}^{(\lambda, \theta)}} \leq\left\|{ }_{0}^{C} D_{t}^{\alpha_{l}} f-{ }_{0}^{C} D_{t}^{\alpha_{l}} u\right\|_{w_{t_{f}}^{(\lambda, \theta, \vartheta)}}, \quad \forall u(t) \in \Lambda_{N} .
$$

Considering the generalized Taylor formula $\left.{ }_{0}^{C} D_{t}^{\alpha_{l}} u(t)=\sum_{j=0}^{N} \frac{t^{j \lambda}}{\Gamma(j \lambda+1)}{ }_{0}^{C} D_{t}^{j \lambda+\alpha_{l}} u\right)\left(0^{+}\right)$yields

$$
\begin{aligned}
\left|{ }_{0}^{C} D_{t}^{\alpha_{l}} f(t)-{ }_{0}^{C} D_{t}^{\alpha_{l}} u(t)\right| & =\left|{ }_{0}^{C} D_{t}^{\alpha_{l}} f(t)-\sum_{j=0}^{N} \frac{t^{j \lambda}}{\Gamma(j \lambda+1)}\left({ }_{0}^{C} D_{t}^{j \lambda+\alpha_{l}} u\right)\left(0^{+}\right)\right| \\
& \leq \mathcal{L}_{l} \frac{t^{(N+1) \lambda}}{\Gamma((N+1) \lambda+1)} .
\end{aligned}
$$

Taking $L_{w_{t_{f}}^{(\lambda, \theta)}}^{2(\lambda, \vartheta)}$-norm in both sides of inequality (21) leads to

$$
\begin{aligned}
\left\|{ }_{0}^{C} D_{t}^{\alpha_{l}} f-{ }_{0}^{C} D_{t}^{\alpha_{l}} u\right\|_{w_{t_{f}}^{(\lambda, \theta, \vartheta)}}^{2} & \leq \frac{\mathcal{L}_{l}^{2}}{(\Gamma((N+1) \lambda+1))^{2}} \int_{0}^{t_{f}} t^{2(N+1) \lambda} w_{t_{f}}^{(\lambda, \theta, \vartheta)} d t \\
& =\frac{\mathcal{L}_{l}^{2}}{(\Gamma((N+1) \lambda+1))^{2}} \lambda \int_{0}^{t_{f}} t^{\lambda(2 N+3+\vartheta)-1}\left(t_{f}^{\lambda}-t^{\lambda}\right)^{\theta} d t .
\end{aligned}
$$


From (17), (18), and (22), we can write

$$
\begin{aligned}
\left\|{ }_{0}^{C} D_{t}^{\alpha_{l}} f-{ }_{0}^{C} D_{t}^{\alpha} u\right\|_{w_{t_{f}}^{(\lambda, \theta, \vartheta)}}^{2} & \leq \frac{\mathcal{L}_{l}^{2}}{(\Gamma((N+1) \lambda+1))^{2}} \int_{0}^{t_{f}} t^{2(N+1) \lambda} w_{t_{f}}^{(\lambda, \theta, \vartheta)} d t \\
& =\frac{\mathcal{L}_{l}^{2} t_{f}^{(2 N+3+\vartheta+\theta) \lambda} \Gamma(1+\theta) \Gamma(2 N+3+\vartheta)}{(\Gamma((N+1) \lambda+1))^{2} \Gamma(4+2 N+\theta+\vartheta)} .
\end{aligned}
$$

Now we take the square root of both sides of (23), and therefore inequality (20) can be obtained.

Theorem 5.6 Let ${ }_{0}^{C} D_{t}^{j \lambda} f(t),{ }_{0}^{C} D_{t}^{j \lambda+q} f(t),{ }_{0}^{C} D_{t}^{j \lambda+\alpha} f(t) \in C\left(\left[0, t_{f}\right]\right)$. Suppose that $\left|{ }_{0}^{C} D_{t}^{j \lambda} f(t)\right| \leq \mathcal{L}$, $\left|{ }_{0}^{C} D_{t}^{j \lambda+q} f(t)\right| \leq \mathcal{L}_{q},\left.\right|_{0} ^{C} D_{t}^{j \lambda+\alpha_{l}} f(t) \mid \leq \mathcal{L}_{l}$, for $l=1,2, \ldots, r$. In Eq. (1), let $G_{1}$ be Lipschitz with the constant $\mu$. Therefore, the error bound of the SFOJPs method for the modified equation is

$$
\left\|E_{k}^{M}\right\|_{w_{t_{f}}^{(\lambda, \theta, \vartheta)}} \leq \frac{(\mu(\beta-\alpha)+(r+1) \kappa) \rho}{\Gamma((N+1) \lambda+1)} \sqrt{\frac{t_{f}^{(2 N+3+\vartheta+\theta) \lambda} \Gamma(1+\theta) \Gamma(2 N+3+\vartheta)}{\Gamma(4+2 N+\theta+\vartheta)}},
$$

where $\kappa=\max _{j=0, \ldots, r}\left\{\kappa_{j}\right\}$ and $\rho=\max _{l=1, \ldots, r}\left\{\mathcal{L}, \mathcal{L}_{q}, \mathcal{L}_{l}\right\}$.

Proof Since $G_{1}$ is Lipschitz with the constant $\mu$, we can write

$$
\begin{aligned}
& \left|E_{N}\right|=\mid \int_{\alpha}^{\beta} G_{1}\left(q,\left({ }_{0}^{C} D_{t}^{q} f\right)_{N}(t)\right) d q \\
& +G_{2}\left(t, f_{N}(t),\left({ }_{0}^{C} D_{t}^{\alpha_{1}} f\right)_{N}(t), \ldots,\left({ }_{0}^{C} D_{t}^{\alpha_{r}} f\right)_{N}(t)\right)-g(t) \\
& =\mid \int_{\alpha}^{\beta} G_{1}\left(q,\left({ }_{0}^{C} D_{t}^{q} f\right)_{N}(t)\right) d q+G_{2}\left(t, f_{N}(t),\left({ }_{0}^{C} D_{t}^{\alpha_{1}} f\right)_{N}(t), \ldots,\left({ }_{0}^{C} D_{t}^{\alpha_{r}} f\right)_{N}(t)\right) \\
& -\int_{\alpha}^{\beta} G_{1}\left(q,{ }_{0}^{C} D_{t}^{q} f(t)\right) d q-G_{2}\left(t, f(t),{ }_{0}^{C} D_{t}^{\alpha_{1}} f(t), \ldots,{ }_{0}^{C} D_{t}^{\alpha_{r}} f(t)\right) \\
& \leq \mu \int_{\alpha}^{\beta}\left|{ }_{0}^{C} D_{t}^{q} f(t)-\left({ }_{0}^{C} D_{t}^{q} f\right)_{N}(t)\right| d q+\kappa\left|f(t)-f_{N}(t)\right| \\
& +\kappa\left|{ }_{0}^{C} D_{t}^{\alpha_{1}} f(t)-\left({ }_{0}^{C} D_{t}^{\alpha_{1}} f\right)_{N}(t)\right|+\cdots+\kappa\left|{ }_{0}^{C} D_{t}^{\alpha_{r}} f(t)-\left({ }_{0}^{C} D_{t}^{\alpha_{r}} f\right)_{N}(t)\right| \\
& \leq \mu \int_{\alpha}^{\beta}\left\|{ }_{0}^{C} D_{t}^{q} f-\left({ }_{0}^{C} D_{t}^{q} f\right)_{N}\right\|_{w_{t_{f}}^{(\lambda, \theta, \vartheta)}} d q+\kappa\left\|f-f_{N}\right\|_{w_{t_{f}}^{(\lambda, \theta, \vartheta)}} \\
& +\kappa\left\|_{0}^{C} D_{t}^{\alpha_{1}} f-\left({ }_{0}^{C} D_{t}^{\alpha_{1}} f\right)_{N}\right\|_{w_{t_{f}}^{(\lambda, \theta, \vartheta)}}+\cdots+\kappa\left\|{ }_{0}^{C} D_{t}^{\alpha_{r}} f-\left({ }_{0}^{C} D_{t}^{\alpha_{r}} f\right)_{N}\right\|_{w_{t_{f}}^{(\lambda, \theta, \vartheta)}} \text {. }
\end{aligned}
$$

By using Theorems 5.4 and 5.5 , we obtain

$$
\left\|E_{N}\right\|_{w_{t_{f}}^{(\lambda, \theta, \vartheta)}} \leq \frac{\mu(\beta-\alpha) \mathcal{L}_{q}+\kappa\left(\mathcal{L}+\mathcal{L}_{1}+\cdots+\mathcal{L}_{r}\right)}{\Gamma((N+1) \lambda+1)} \sqrt{\frac{t_{f}^{(2 N+3+\vartheta+\theta) \lambda} \Gamma(1+\theta) \Gamma(2 N+3+\vartheta)}{\Gamma(4+2 N+\theta+\vartheta)}} .
$$

Let

$$
\rho=\max _{l=1, \ldots, r}\left\{\mathcal{L}, \mathcal{L}_{q}, \mathcal{L}_{l}\right\} .
$$


Table 1 Absolute errors at the interval $[0,1)$ with $M=3$, for Example 6.1

\begin{tabular}{lll}
\hline$t$ & \multicolumn{1}{l}{ SKCWs } & \\
\cline { 2 - 3 } & $k=1, \hat{m}=6$ & $k=2, \hat{m}=12$ \\
\hline 0.1 & $3.294606 \mathrm{e}-4$ & $1.549570 \mathrm{e}-5$ \\
0.2 & $4.790596 \mathrm{e}-4$ & $2.219229 \mathrm{e}-4$ \\
0.3 & $2.300451 \mathrm{e}-3$ & $1.478095 \mathrm{e}-5$ \\
0.4 & $1.480546 \mathrm{e}-3$ & $2.725903 \mathrm{e}-4$ \\
0.5 & $1.591482 \mathrm{e}-2$ & $1.821096 \mathrm{e}-3$ \\
0.6 & $1.669851 \mathrm{e}-3$ & $3.456476 \mathrm{e}-4$ \\
0.7 & $2.919086 \mathrm{e}-3$ & $4.227610 \mathrm{e}-5$ \\
0.8 & $1.593268 \mathrm{e}-3$ & $3.777966 \mathrm{e}-4$ \\
0.9 & $5.069659 \mathrm{e}-4$ & $1.134724 \mathrm{e}-4$ \\
\hline
\end{tabular}

Therefore, we get

$$
\left\|E_{N}\right\|_{w_{t_{f}}^{(\lambda, \theta, \vartheta)}} \leq \frac{(\mu(\beta-\alpha)+(r+1) \kappa) \rho}{\Gamma((N+1) \lambda+1)} \sqrt{\frac{t_{f}^{(2 N+3+\vartheta+\theta) \lambda} \Gamma(1+\theta) \Gamma(2 N+3+\vartheta)}{\Gamma(4+2 N+\theta+\vartheta)}} .
$$

\section{Illustrative examples}

In this section, we present five problems which are tested by Maple 2018. Also, we obtain the absolute errors by

$$
\left|f(t)-f_{k, M}(t)\right| \text { and } \quad\left|f(t)-f_{N}(t)\right|, \quad t \in\left[0, t_{f}\right), k, M, N \in \mathbb{N} \text {. }
$$

Note that, in all tables, $\hat{m}$ denotes the numbers of bases.

Example 6.1 Consider the following nonlinear DOFDE:

$$
\begin{aligned}
& \int_{0}^{3}\left(\frac{\Gamma(4.5-q)}{105 \sqrt{\pi}}{ }_{0}^{C} D_{t}^{q} f(t)\right)^{\frac{1}{2}} d q=\frac{\sqrt[4]{t}(t \sqrt{t}-1)}{2 \ln (t)}, \\
& f(0)=f^{\prime}(0)=f^{\prime \prime}(0)=0
\end{aligned}
$$

with the exact solution $f(t)=t^{3} \sqrt{t}$.

In the above problem, the distributed-order term is discretized with the seven-point Gauss-Legendre quadrature rule. The numerical results at the interval $[0,1)$, obtained by the SKCWs method, are reported in Table 1 by selecting $\hat{m}=2^{k} M=6,12$. Graphs of the exact and approximate solutions and also absolute errors, obtained by the mentioned method with $k=2, M=3$, are plotted in Fig. 1 . This figure and Table 1 illustrate the efficiency and accuracy of the method.

Example 6.2 Consider the DOFDE in the following form [14, 19, 21, 39]:

$$
\begin{aligned}
& \int_{0}^{2} \frac{\Gamma(6-q)}{120}{ }_{0}^{C} D_{t}^{q} f(t) d q=\frac{t^{5}-t^{3}}{\ln t}, \\
& f(0)=f^{\prime}(0)=0
\end{aligned}
$$

with the exact solution $f(t)=t^{5}$. 


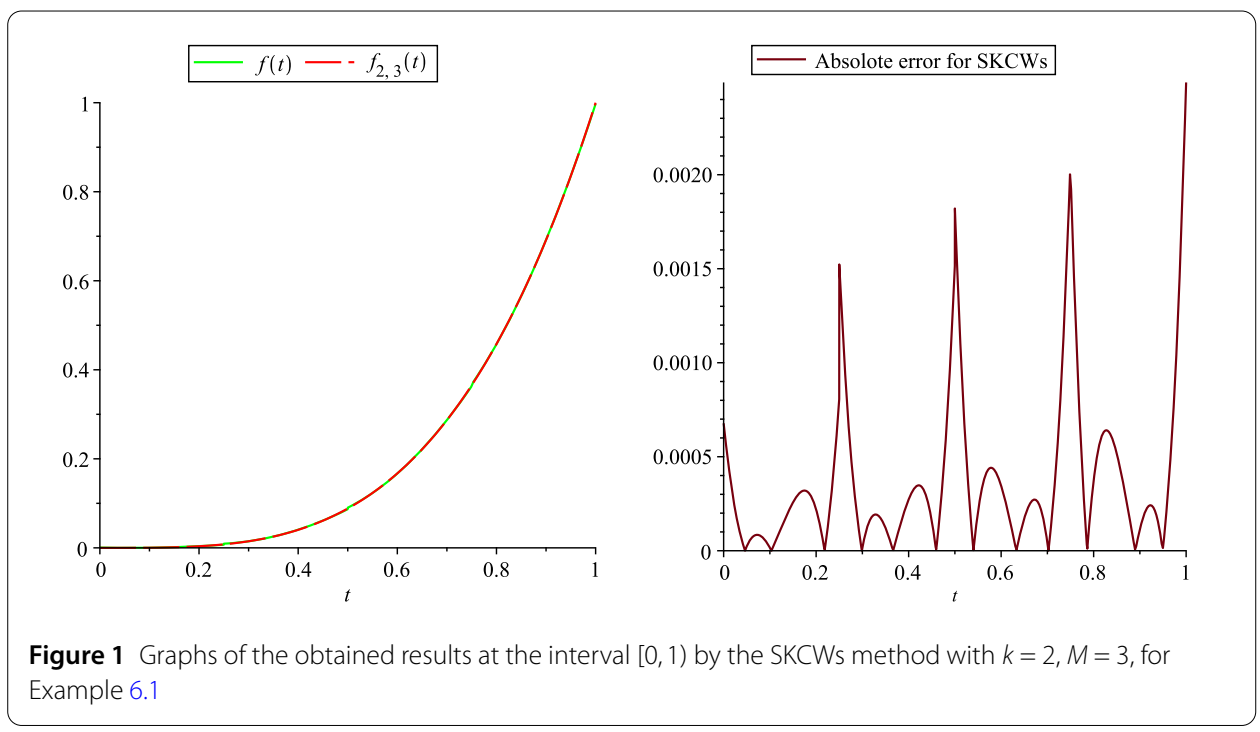

Table 2 Absolute errors at the interval $[0,1)$, for Example 6.2

\begin{tabular}{|c|c|c|c|c|c|c|}
\hline \multirow[t]{2}{*}{$t$} & \multicolumn{2}{|c|}{ 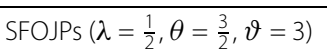 } & \multicolumn{2}{|l|}{ SKCWs } & \multirow{2}{*}{$\begin{array}{l}\text { HLBP [39] } \\
M=3, N=8, \hat{m}=24\end{array}$} & \multirow{2}{*}{$\begin{array}{l}\text { BPFs [39] } \\
\overline{N=32, \hat{m}=32}\end{array}$} \\
\hline & $\bar{N}=8, \hat{m}=9$ & $N=11, \hat{m}=12$ & $\hat{k}=1, M=8, \hat{m}=16$ & $k=1, M=10, \hat{m}=20$ & & \\
\hline$\overline{0.1}$ & $9.548407 \mathrm{e}-6$ & $1.047112 \mathrm{e}^{-12}$ & $3.937368 \mathrm{e}-6$ & $2.322781 \mathrm{e}-6$ & $4.4538 \mathrm{e}^{-7}$ & $5.9470 \mathrm{e}-6$ \\
\hline 0.2 & $6.250375 e-6$ & $1.531553 \mathrm{e}^{-12}$ & $2.021002 e^{-5}$ & $1.209247 \mathrm{e}-5$ & $1.3319 \mathrm{e}-5$ & $1.3073 e^{-5}$ \\
\hline 0.3 & $1.705950 e^{-}-7$ & $1.176192 \mathrm{e}^{-12}$ & $5.028661 \mathrm{e}-5$ & $3.032198 \mathrm{e}-5$ & $8.6726 \mathrm{e}-5$ & $1.9812 \mathrm{e}^{-4}$ \\
\hline 0.4 & $6.318599 \mathrm{e}-6$ & $1.094817 \mathrm{e}-12$ & $9.335682 \mathrm{e}-5$ & $5.654374 \mathrm{e}-5$ & $2.6389 e^{-4}$ & $1.4000 e^{-3}$ \\
\hline 0.5 & $4.719573 e-6$ & $1.183008 \mathrm{e}^{-12}$ & $1.479691 \mathrm{e}-4$ & $8.987633 e^{-5}$ & $5.5607 \mathrm{e}^{-4}$ & $4.7000 e^{-3}$ \\
\hline 0.6 & $4.864510 \mathrm{e}-6$ & $7.238237 \mathrm{e}^{-13}$ & $2.126070 \mathrm{e}-4$ & $1.293900 \mathrm{e}-4$ & $9.2704 e^{-4}$ & $5.4000 e^{-3}$ \\
\hline 0.7 & $9.045018 \mathrm{e}-6$ & $6.241959 \mathrm{e}^{-13}$ & $2.858959 \mathrm{e}-4$ & $1.742388 \mathrm{e}-4$ & $1.3000 \mathrm{e}-3$ & $2.7000 e^{-3}$ \\
\hline 0.8 & $1.511866 e^{-7}$ & $9.814239 \mathrm{e}^{-13}$ & $3.666613 \mathrm{e}-4$ & $2.236985 e^{-4}$ & $1.6000 \mathrm{e}-3$ & $7.6000 e^{-3}$ \\
\hline 0.9 & $1.864086 \mathrm{e}-5$ & $3.293418 \mathrm{e}-14$ & $4.539275 e^{-4}$ & $2.771667 \mathrm{e}-4$ & $1.6000 \mathrm{e}-3$ & $3.1300 \mathrm{e}-2$ \\
\hline
\end{tabular}

In the above problem, we discretize the distributed-order term with the eight-point Gauss-Legendre quadrature rule. In Table 2, we report the absolute errors at the interval $[0,1)$ for the SKCWs method by selecting $\hat{m}=N+1=9,12$ with $\lambda=\frac{1}{2}, \theta=\frac{3}{2}, \vartheta=3$; for the SFOJPs method by selecting $\hat{m}=2^{k} M=16,20$; for the hybrid of Legendre polynomials and block-pulse functions (HLBP) method [39] by selecting $\hat{m}=N M=24$; and for the block-pulse functions (BPFs) method [39] by selecting $\hat{m}=N=32$. Graphs of the exact and approximate solutions and also absolute errors, obtained by the SKCWs and SFOJPs methods with $k=1, M=10, N=11, \lambda=\frac{1}{2}, \theta=\frac{3}{2}, \vartheta=3$, are plotted in Fig. 2 . This figure and Table 2 show the efficiency and accuracy of the new methods in comparison with the other methods reported in [39].

Example 6.3 Consider the DOFDE in the following form [19-21, 38, 50, 53]:

$$
\begin{gathered}
f^{\prime \prime}(t)+\omega^{2} f(t)+\sigma(t)=g(t), \quad f(0)=f^{\prime}(0)=0, \\
\int_{0}^{1} a^{q}{ }_{0}{ }^{C} D_{t}^{q} \sigma(t) d q=\gamma \int_{0}^{1} b_{0}^{q C} D_{t}^{q} f(t) d q .
\end{gathered}
$$

The above equations describe the motion of the oscillator, where $\gamma, a, b$ are constants; $\omega$ is the eigen frequency of the undamped system; $g(t)$ is the external forcing function; $f(t)$ 


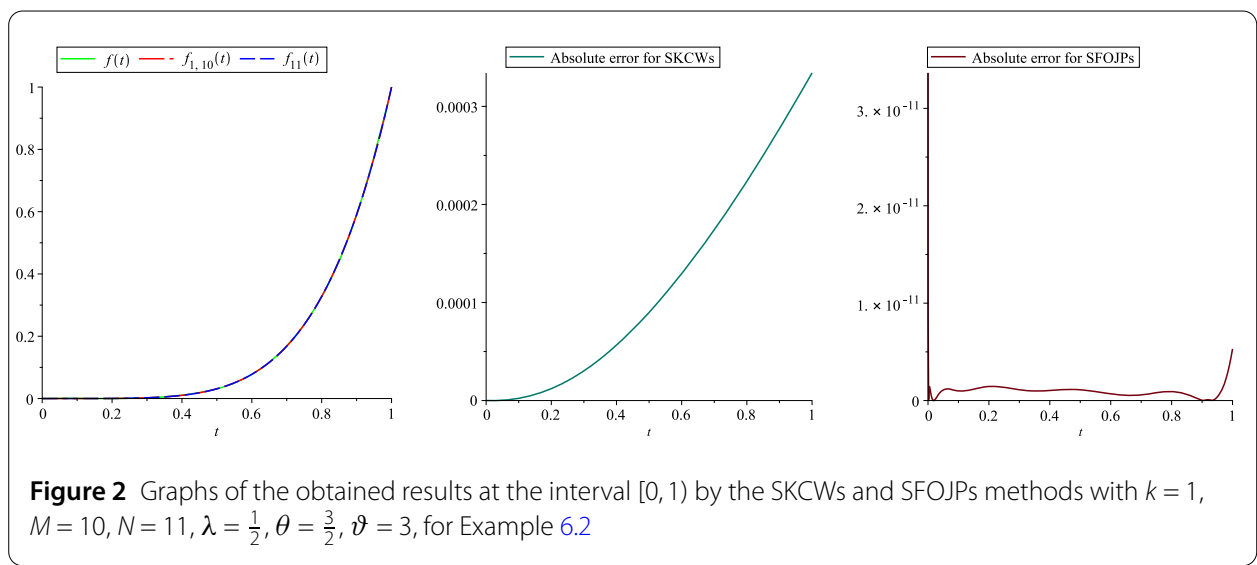

Table 3 Absolute errors at the interval [0,10), for Example 6.3

\begin{tabular}{|c|c|c|c|c|}
\hline \multirow[t]{2}{*}{$t$} & \multicolumn{2}{|c|}{ SFOJPS $\left(\lambda=\frac{1}{2}, \theta=\vartheta=0\right)$} & \multirow{2}{*}{$\begin{array}{l}\text { SKCWs [47] } \\
k=2, M=13, \hat{m}=52\end{array}$} & \multirow{2}{*}{$\frac{\operatorname{MLWs}(v=1)[36]}{k=3, M=15, \hat{m}=60}$} \\
\hline & $N=39, \hat{m}=40$ & $N=49, \hat{m}=50$ & & \\
\hline 1 & $3.565546 \mathrm{e}-6$ & $4.583174 \mathrm{e}^{-10}$ & $1.320582 e^{-}-7$ & $4.581149 e^{-9}$ \\
\hline 2 & $1.609513 \mathrm{e}-6$ & $2.347220 \mathrm{e}-10$ & $4.245111 \mathrm{e}^{-7}$ & $1.822323 e^{-}-8$ \\
\hline 3 & $3.562010 \mathrm{e}-6$ & $1.458788 \mathrm{e}-10$ & $2.178349 e^{-5}$ & $1.097018 \mathrm{e}-6$ \\
\hline 4 & $5.346519 e^{-7}$ & $1.887288 \mathrm{e}^{-10}$ & $2.208436 \mathrm{e}-5$ & $1.110414 \mathrm{e}-6$ \\
\hline 5 & $4.216773 e^{-}-7$ & $5.229296 \mathrm{e}^{-10}$ & $2.241317 \mathrm{e}-5$ & $1.121390 \mathrm{e}-6$ \\
\hline 6 & $3.034043 \mathrm{e}^{-7}$ & $8.984458 e^{-11}$ & $2.196957 \mathrm{e}-5$ & $1.106670 \mathrm{e}-6$ \\
\hline 7 & $8.445049 e^{-7}$ & $4.972243 e^{-10}$ & $2.118251 \mathrm{e}-5$ & $1.069842 \mathrm{e}-6$ \\
\hline 8 & $3.841176 \mathrm{e}-6$ & $3.062962 \mathrm{e}^{-10}$ & $5.110080 e^{-5}$ & $2.290284 \mathrm{e}-6$ \\
\hline 9 & $1.904467 \mathrm{e}-6$ & $4.402973 \mathrm{e}^{-10}$ & $5.188183 e^{-5}$ & $2.326670 \mathrm{e}-6$ \\
\hline
\end{tabular}

and $\sigma(t)$ are the displacement and the dissipation force, respectively. In this problem, the forced vibrations of the distributed-order oscillator subjected to the harmonic excitation $g(t)=g_{0} \sin (\Omega t)$ are studied. The solution of this problem is obtained with $g_{0}=1, \Omega=1.2 \omega$, $\omega=3$, and $\gamma=1$. If $a=b$, the solution is identical to the elastic with $\omega_{e l}=\sqrt{1+\omega^{2}}=\sqrt{10}$, and the exact solution is

$$
f(t)=\frac{g_{0}}{\omega_{e l}^{2}-\Omega^{2}}\left(\sin \Omega t-\frac{\Omega}{\omega_{e l}} \sin \omega_{e l} t\right)
$$

In Table 3, we report the absolute errors at the interval $[0,10)$ for the SFOJPs method by selecting $\hat{m}=N+1=39,49$ with $\lambda=\frac{1}{2}, \theta=\vartheta=0$; for the second kind Chebyshev wavelets (SKCWs) method [47] by selecting $\hat{m}=N(M+1)=52$; and for the Müntz-Legendre wavelets (MLWs) method [36] by selecting $\hat{m}=N(M+1)=60$. We emphasize that the results reported in [36, 47] have been compared with the results reported in [19, 38, 39, 53], and it was concluded that the methods in $[36,47]$ are more accurate than the other methods. Therefore, here, we just compare our method with the methods of [36, 47]. Graphs of the exact and approximate solutions and also absolute errors, obtained by the SKCWs and SFOJPs with $k=4, M=5, N=49, \lambda=\frac{1}{2}, \theta=\vartheta=0$, are plotted in Fig. 3. This figure and Table 3 show the efficiency and accuracy of the SFOJPs method in comparison with the methods reported in $[36,47]$. 


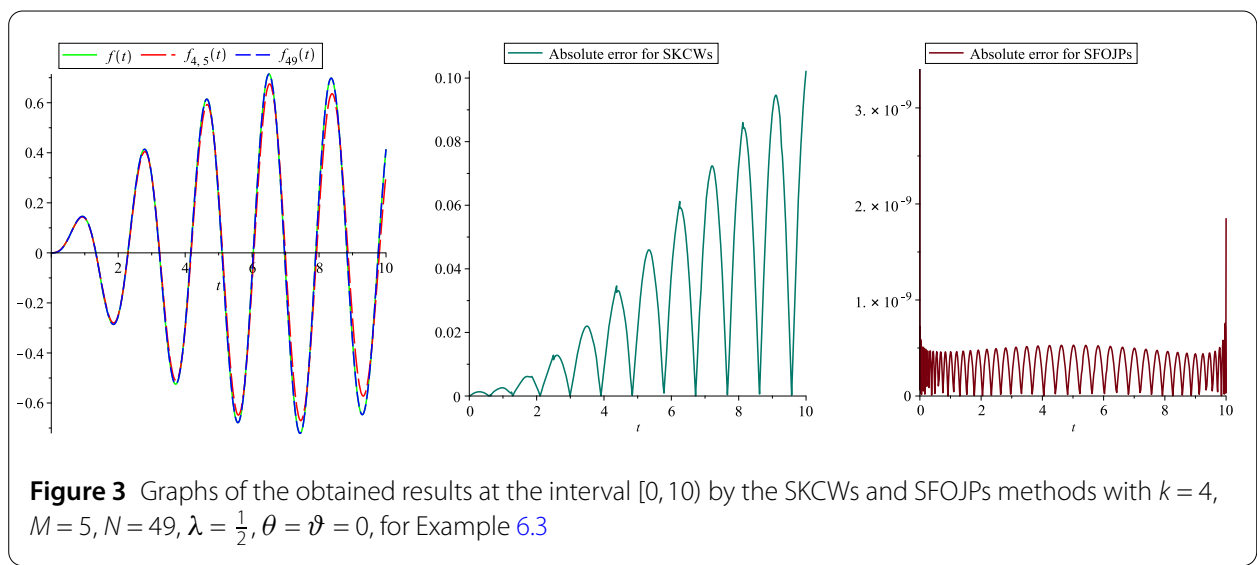

Table 4 Absolute errors at the interval $[0,10)$, for Example 6.4

\begin{tabular}{llll}
\hline$s$ & $\operatorname{SFOJPS}\left(\lambda=\frac{1}{2}, \theta=\frac{3}{2}, \vartheta=3\right)$ & & MLWs $(\nu=1)[36]$ \\
\cline { 2 - 4 } & $N=3, \hat{m}=4$ & $N=8, \hat{m}=9$ & $k=4, M=2, \hat{m}=16$ \\
\hline 2 & $3.144616 \mathrm{e}-3$ & $2.097341 \mathrm{e}-4$ & $3.514551 \mathrm{e}-3$ \\
3 & $2.531630 \mathrm{e}-3$ & $1.746139 \mathrm{e}-4$ & $2.757827 \mathrm{e}-3$ \\
4 & $2.143702 \mathrm{e}-3$ & $1.533629 \mathrm{e}-4$ & $2.286212 \mathrm{e}-3$ \\
5 & $1.871068 \mathrm{e}-3$ & $1.388309 \mathrm{e}-4$ & $1.941673 \mathrm{e}-3$ \\
6 & $1.667009 \mathrm{e}-3$ & $1.281428 \mathrm{e}-4$ & $1.673715 \mathrm{e}-3$ \\
7 & $1.507569 \mathrm{e}-3$ & $1.198746 \mathrm{e}-4$ & $1.459252 \mathrm{e}-3$ \\
8 & $1.379004 \mathrm{e}-3$ & $1.132319 \mathrm{e}-4$ & $1.284616 \mathrm{e}-3$ \\
9 & $1.272802 \mathrm{e}-3$ & $1.077350 \mathrm{e}-4$ & $1.140532 \mathrm{e}-3$ \\
\hline
\end{tabular}

Example 6.4 Consider the following distributed-order fractional relaxation equation [18, 31, 50]:

$$
\begin{aligned}
& { }_{0}^{C} D_{t}^{p(q)} f(t)+0.1 f(t)=0, \quad p(q)=6 q(1-q), \quad 0 \leq q \leq 1, \\
& f(0)=1,
\end{aligned}
$$

with the exact solution [31]

$$
F(s)=\mathscr{L}\{f(t)\}=\frac{\Theta(s) / s}{0.1+\Theta(s)},
$$

in the Laplace domain, where

$$
\Theta(s)=\frac{\ln (s)(6 s+6)-12 s+12}{(\ln (s))^{3}} .
$$

In the above problem, we discretize the distributed-order term with the three-point Gauss-Legendre quadrature rule. In Table 4, we report the absolute errors at the interval $[0,10)$ for the SFOJPs method by selecting $\hat{m}=N+1=4,9$ with $\lambda=\frac{1}{2}, \theta=\frac{3}{2}, \vartheta=3$; and for the Müntz-Legendre wavelets (MLWs) method [36] with $\hat{m}=2^{k-1} M=16,32$. Figure 4 shows that by using 8 number of SFOJPs the obtained results are better than the results of [50] that obtained by using $1000 \mathrm{BPFs}$ for solving this problem. 

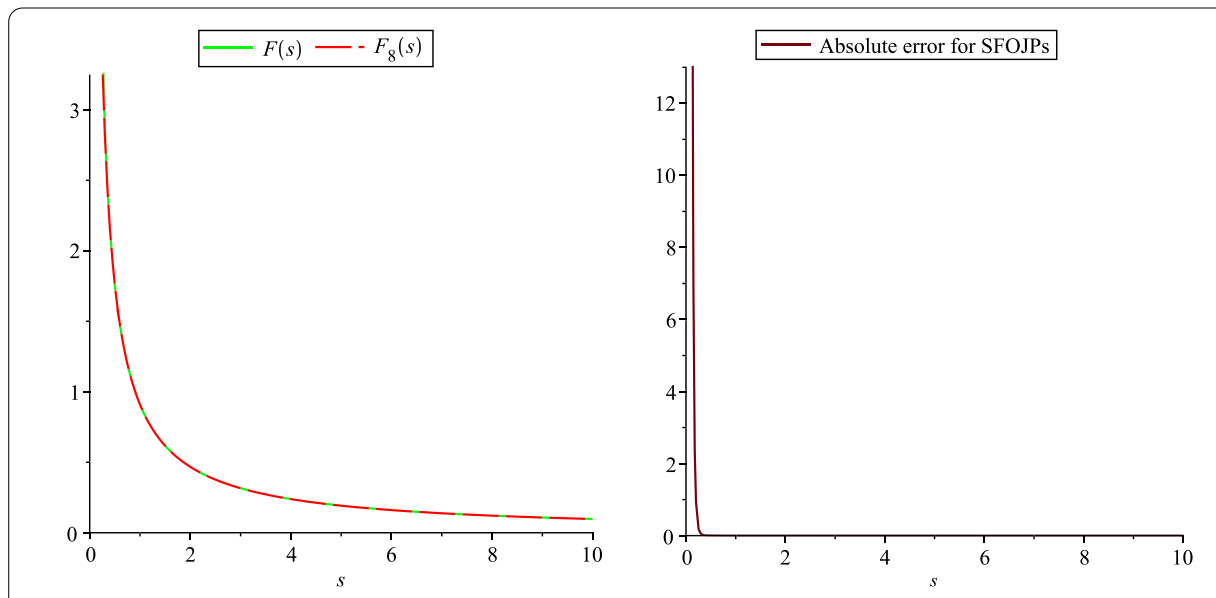

Figure 4 Graphs of the obtained results at the interval $[0,10)$ by the SFOJPs method with $N=8, \lambda=\frac{1}{2}, \theta=\frac{3}{2}$, $\vartheta=3$, for Example 6.4

Figure 5 The approximate solution at the interval [0, 30) by the SKCWs method with $k=5, M=3$, for Example 6.5

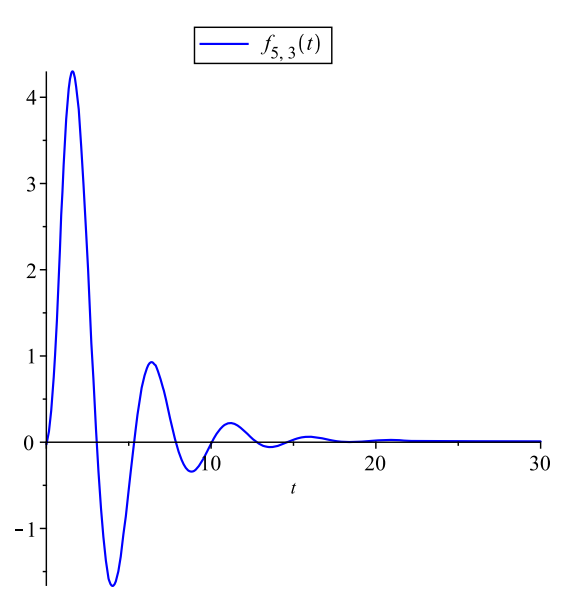

Example 6.5 Consider the following Bagley-Torvik equation [7, 8], where the damping term is expressed in terms of distributed-order derivatives [46]:

$$
\begin{aligned}
& a f^{\prime \prime}(t)+b_{0}^{C} D_{t}^{p(q)} f(t)+c f(t)=\left\{\begin{array}{ll}
8, & 0 \leq t \leq 1, \\
0, & t>1,
\end{array} \quad p(q)=6 q(1-q), 0 \leq q \leq 1,\right. \\
& f(0)=0, f^{\prime}(0)=0 .
\end{aligned}
$$

This equation is called fractional oscillator equation, when the order of damping term is constant.

In the above problem, the distributed-order term is discretized with the three-point Gauss-Legendre quadrature rule. The graph of the approximate solution at the interval $[0,30)$ is plotted in Fig. 5 by using the SKCWs method with $k=5, M=3, a=b=c=1$. This figure has a good agreement with Fig. 8, reported in [46]. 


\title{
7 Conclusion
}

In this research paper, based on Schauder's and Tychonoff's fixed point theorems, sufficient conditions for the local and global existence of solutions were provided for general DOFDEs. Also, sufficient conditions were provided for the uniqueness of the solutions. Furthermore, we proposed new methods to solve DOFDEs of the general form in the time domain. By using these methods, the mentioned equations were reduced to systems of algebraic equations. We solved these systems by using the "fsolve" command of Maple 2018. The error bounds of the methods have been discussed. In addition, the presented methods were implemented for two test problems and some famous distributed-order models, such as the model that describes the motion of the oscillator, the distributed-order fractional relaxation equation, and the Bagley-Torvik equation. It showed that by applying the SKCWs and SFOJPs methods, the obtained results are better than the other existing methods. We deduce that the proposed methods are efficient numerical tools for solving DOFDEs.

\author{
Acknowledgements \\ Not applicable. \\ Funding \\ Not applicable. \\ Availability of data and materials \\ Not applicable.
}

\section{Declarations}

Ethics approval and consent to participate

Not applicable.

Consent for publication

The authors confirm that the work described has not been published before and that its publication has been approved by all co-authors.

\section{Competing interests}

The authors declare that they have no competing interests.

Authors' contributions

All authors have contributed equally to this manuscript. All authors read and approved the final manuscript.

Authors' information

Not applicable.

\section{Publisher's Note}

Springer Nature remains neutral with regard to jurisdictional claims in published maps and institutional affiliations.

Received: 14 June 2021 Accepted: 24 September 2021 Published online: 18 October 2021

References

1. Ali, K.K., Abd El Salam, M.A., Mohamed, E.M.H., Samet, B., Kumar, S., Osman, M.S.: Numerical solution for generalized nonlinear fractional integro-differential equations with linear functional arguments using Chebyshev series. Adv. Differ. Equ. 2020, 494 (2020). https://doi.org/10.1186/s13662-020-02951-z

2. Ali, K.K., Osman, M.S., Baskonus, H.M., Elazabb, N.S., Illhan, E.: Analytical and numerical study of the HIV-1 infection of $\mathrm{CD}^{+} \mathrm{T}$-cells conformable fractional mathematical model that causes acquired immunodeficiency syndrome with the effect of antiviral drug therapy. Math. Methods Appl. Sci. (2020). https://doi.org/10.1002/mma.7022

3. Arqub, O.A., Osman, M.S., Abdel-Ayat, A., Mohamed, A.A., Momani, S.: A numerical algorithm for the solutions of ABC singular Lane-Emden type models arising in astrophysics using reproducing kernel discretization method. Mathematics 8(6), 923 (2020). https://doi.org/10.3390/math8060923

4. Atanacković, T.M.: A generalized model for the uniaxial isothermal deformation of a viscoelastic body. Acta Mech. $159,77-86(2002)$

5. Atanacković, T.M., Oparnica, L., Pilipović, S.: On a nonlinear distributed order fractional differential equation. J. Math. Anal. Appl. 328, 590-608 (2007) 
6. Bagley, R.L., Torvik, P.J.: Fractional calculus in the transient analysis of viscoelastically damped structures. AIAA J. 23, 918-925 (1985)

7. Bagley, R.L., Torvik, P.J.: On the existence of the order domain and the solution of distributed order equations-part I. Int. J. Appl. Math. 2, 865-882 (2000)

8. Bagley, R.L., Torvik, P.J.: On the existence of the order domain and the solution of distributed order equations-part II. Int. J. Appl. Math. 2, 965-988 (2000)

9. Canuto, C., Hussaini, M.Y., Quarteroni, A., Zang, T.A.: Spectral Methods: Fundamentals in Single Domains. Springer, New York (2006)

10. Caputo, M.: Mean-fractional-order-derivative differential equation and filters. Ann. Univ. Ferrara 41, 73-84 (1995)

11. Chechkin, A.V., Gorenflo, R., Sokolov, I.M., Gonchar, V.Y.: Distributed order time fractional diffusion equation. Fract. Calc. Appl. Anal. 6, 259-279 (2003)

12. Conway, J.B.: A Course in Functional Analysis. Springer, Berlin (2007)

13. Cuahutenango-Barro, B., Taneco-Hernández, M.A., Lv, Y.P., Gómez-Aguilar, J.F., Osman, M.S., Jahanshahi, H., Aly, A.A.: Analytical solutions of fractional wave equation with memory effect using the fractional derivative with exponential kernel. Results Phys. 25, 104148 (2021). https://doi.org/10.1016/j.rinp.2021.104148

14. Diethelm, K., Ford, N.J: Numerical analysis for distributed-order differential equation. J. Comput. Appl. Math. 225(1), 96-104 (2009)

15. Diennadi, S., Shawagfeh, N., Inc, M., Osman, M.S., Gómez-Aguilar, J.F., Arqub, O.A.: The Tikhonov regularization method for the inverse source problem of time fractional heat equation in the view of $A B C$-fractional technique. Phys. Scr. 96(9), 094006 (2021). https://doi.org/10.1088/1402-4896/ac0867

16. Gorenflo, R., Mainardi, F., Scalas, E., Raberto, M.: Fractional calculus and continuous-time finance III: the diffusion limit. In: Mathematical Finance, pp. 171-180. Springer, Berlin (2001)

17. Höfling, F., Franosch, T.: Anomalous transport in the crowded world of biological cells. Rep. Prog. Phys. 76(4), 046602 (2013)

18. Jiao, Z., Chen, Y.Q., Podlubny, I.: Distributed Order Dynamic System Stability. Simulation and Perspective. Springer, London (2012)

19. Jibenja, N., Yuttanan, B., Razzaghi, M.: An efficient method for numerical solutions of distributed order fractional differential equations. J. Comput. Nonlinear Dyn. 13,1-11 (2018)

20. Katsikadelis, J.T.: Fractional distributed order oscillator: a numerical solution. J. Serb. Soc. Comput. Mech. 6, 148-159 (2012)

21. Katsikadelis, J.T: Numerical solution of distributed order fractional differential equations. J. Comput. Phys. 259, 11-22 (2014)

22. Kayedi-Bardeh, A., Eslahchi, M.R., Dehghan, M.: A method for obtaining the operational matrix of fractional Jacobi functions and applications. J. Vib. Control 20(5), 736-748 (2014)

23. Kilıçman, A., Al Zhour, Z.A.A.: Kronecker operational matrices for fractional calculus and some applications. Appl. Math. Comput. 187(1), 250-265 (2007)

24. Kochubei, A.N.: Distributed order calculus and equations of ultraslow diffusion (2007). arXiv preprint math-ph/0703046

25. Kumar, S., Kumar, R., Osman, M.S., Samet, B.: A wavelet based numerical scheme for fractional order SEIR epidemic of measles by using Genocchi polynomials. Numer. Methods Partial Differ. Equ. 37(2), 1250-1268 (2021). https://doi.org/10.1002/num.22577

26. Kumar, Y., Singh, S., Srivastava, N., Singh, A., Singh, V.K.: Wavelet approximation scheme for distributed order fractional differential equations. Comput. Math. Appl. 80, 1985-2017 (2020)

27. Li, Y., Sheng, H., Chen, Y.Q.: On distributed order integrator/differentiator. Signal Process. 91, 1079-1084 (2011)

28. Liang, Y., Chen, W., Xu, W., Sun, H.: Distributed order Hausdorff derivative diffusion model to characterize non-Fickian diffusion in porous media. Commun. Nonlinear Sci. Numer. Simul. 70, 384-393 (2019)

29. Liu, D.Y., Tian, Y., Boutat, D., Laleg-Kirati, T.M.: An algebraic fractional order differentiator for a class of signals satisfying a linear differential equation. Signal Process. 116, 78-90 (2015)

30. Magin, R.L.: Fractional calculus models of complex dynamics in biological tissues. Comput. Math. Appl. 59, 1586-1593 (2010)

31. Mainardi, F., Mura, A., Gorenflo, R., Stojanovic, M.: The two form of fractional relaxation of distributed order. J. Vib. Control 13, 1249-1268 (2007)

32. Mainardi, F., Raberto, M., Gorenflo, R., Scalas, E.: Fractional calculus and continuous-time finance ll: the waiting-time distribution. Phys. A, Stat. Mech. Appl. 287, 468-481 (2000)

33. Maleknejad, K., Rashidinia, J., Eftekhari, T.: Numerical solution of three-dimensional Volterra-Fredholm integral equations of the first and second kinds based on Bernstein's approximation. Appl. Math. Comput. 339, 272-285 (2018). https://doi.org/10.1016/j.amc.2018.07.021

34. Maleknejad, K., Rashidinia, J., Eftekhari, T.: Existence, uniqueness, and numerical analysis of solutions for some classes of two-dimensional nonlinear fractional integral equations in a Banach space. Comput. Appl. Math. 39(4), 1-22 (2020). https://doi.org/10.1007/s40314-020-01322-4

35. Maleknejad, K., Rashidinia, J., Eftekhari, T.: Operational matrices based on hybrid functions for solving general nonlinear two-dimensional fractional integro-differential equations. Comput. Appl. Math. 39(2), 1-34 (2020). https://doi.org/10.1007/s40314-020-1126-8

36. Maleknejad, K., Rashidinia, J., Eftekhari, T.: Numerical solutions of distributed order fractional differential equations in the time domain using the Müntz-Legendre wavelets approach. Numer. Methods Partial Differ. Equ. 37(1), 707-731 (2021). https://doi.org/10.1002/num.22548

37. Maleknejad, K., Rashidinia, J., Eftekhari, T.: A new and efficient numerical method based on shifted fractional-order Jacobi operational matrices for solving some classes of two-dimensional nonlinear fractional integral equations. Numer. Methods Partial Differ. Equ. 37, 2687-2713 (2021). https://doi.org/10.1002/num.22762

38. Mashayekhi, S., Razzaghi, M.: Numerical solution of distributed order fractional differential equations by hybrid functions. J. Comput. Phys. 315, 169-181 (2016)

39. Mashoof, M., Refahi Shekhani, A.H.: Simulating the solution of the distributed order fractional differential equations by block-pulse wavelets. UPB Sci. Bull., Ser. A, Appl. Math. Phys. 79, 193-206 (2017) 
40. Meerschaert, M.M., Nane, E., Vellaisamy, P.: Distributed-order fractional diffusions on bounded domains. J. Math. Anal. Appl. 379, 216-228 (2011)

41. Miller, K.S., Ross, B.: An Introduction to the Fractional Calculus and Fractional Differential Equations. Wiley, New York (1993)

42. Naber, M.: Distributed order fractional sub-diffusion. Fractals 12, 23-32 (2014)

43. Nisar, K.S., Ciancio, A., Ali, K.K., Osman, M.S., Cattani, C., Baleanu, D., Zafar, A., Raheel, M., Azeem, M.: On beta-time fractional biological population model with abundant solitary wave structures. Alex. Eng. J. (2021). https://doi.org/10.1016/j.aej.2021.06.106

44. Oldham, K.B.: Fractional differential equations in electrochemistry. Adv. Eng. Softw. 41, 9-12 (2010)

45. Park, C., Nuruddeen, R.I., Ali, K.K., Muhammad, L., Osman, M.S.: Dumitru Baleanu, novel hyperbolic and exponential ansatz methods to the fractional fifth-order Korteweg-de Vries equations. Adv. Differ. Equ. 2020, 627 (2020) https://doi.org/10.1186/s13662-020-03087-w

46. Podlubny, I., Skovranek, T., Vinagre Jara, B.M., Petras, I., Verbitsky, V., Chen, Y.Q.: Matrix approach to discrete fractional calculus-III: non-equidistant grids, variable step length and distributed orders. Philos. Trans. R. Soc. Lond. A 371 20120153 (2013)

47. Rashidinia, J., Eftekhari, T., Maleknejad, K.: A novel operational vector for solving the general form of distributed order fractional differential equations in the time domain based on the second kind Chebyshev wavelets. Numer. Algorithms (2021). https://doi.org/10.1007/s11075-021-01088-8

48. Rashidinia, J., Maleknejad, K., Eftekhari, T.: Numerical solutions of two-dimensional nonlinear fractional Volterra and Fredholm integral equations using shifted Jacobi operational matrices via collocation method. J. King Saud Univ., Sci. 33(1), 1-11 (2021). https://doi.org/10.1016/j.jksus.2020.101244

49. Sun, H.G., Li, Z., Zhang, Y., Chen, W.: Fractional and fractal derivative models for transient anomalous diffusion: model comparison. Chaos Solitons Fractals 102, 346-353 (2017)

50. Trung Duong, P.L., Kwok, E., Lee, M.: Deterministic analysis of distributed order systems using operational matrix. Appl. Math. Model. 40, 1929-1940 (2016)

51. Wang, Y., Fan, Q.: The second kind Chebyshev wavelet method for solving fractional differential equations. Appl. Math. Comput. 218, 8592-8601 (2012)

52. Ye, H., Liu, F., Anh, V., Turner, I.: Numerical analysis for the time distributed-order and Riesz space fractional diffusions on bounded domains. IMA J. Appl. Math. 80, 825-838 (2013)

53. Yuttanan, B., Razzaghi, M.: Legendre wavelets approach for numerical solutions of distributed order fractional differential equations. Appl. Math. Model. (2019). https://doi.org/10.1016/j.apm.2019.01.013

54. Zaky, M.A.: A Legendre collocation method for distributed-order fractional optimal control problems. Nonlinear Dyn. 91, 2667-2681 (2018)

55. Zaky, M.A., Tenreiro Machado, J.A.: On the formulation and numerical simulation of distributed order fractional optimal control. Commun. Nonlinear Sci. Numer. Simul. 52, 177-189 (2017)

56. Zaky, M.A., Tenreiro Machado, J.A.: On the formulation and numerical simulation of distributed-order fractional optimal control problems. Commun. Nonlinear Sci. Numer. Simul. 52, 177-189 (2017)

57. Zeidler, E.: Applied Functional Analysis: Applications to Mathematical Physics. Applied Mathematical Sciences, vol. 108. Springer, New York (1995)

\section{Submit your manuscript to a SpringerOpen ${ }^{\circ}$ journal and benefit from:}

- Convenient online submission

- Rigorous peer review

- Open access: articles freely available online

- High visibility within the field

- Retaining the copyright to your article

Submit your next manuscript at $\boldsymbol{~ s p r i n g e r o p e n . c o m ~}$ 